\title{
Dust and gas emission in the prototypical hot core G29.96-0.02 at sub-arcsecond resolution
}

\author{
H. Beuther ${ }^{1}$, Q. Zhang ${ }^{2}$, E. A. Bergin ${ }^{3}$, T. K. Sridharan ${ }^{2}$, T. R. Hunter ${ }^{4}$, and S. Leurini ${ }^{5}$ \\ 1 Max-Planck-Institute for Astronomy, Königstuhl 17, 69117 Heidelberg, Germany \\ e-mail: beuther@mpia.de \\ 2 Harvard-Smithsonian Center for Astrophysics, 60 Garden Street, Cambridge, MA 02138, USA \\ e-mail: name@cfa.harvard.edu \\ 3 University of Michigan, Dept. of Astronomy, Ann Arbor, MI 48109-1090, USA \\ e-mail: ebergin@umich.edu \\ ${ }^{4}$ NRAO, 520 Edgemont Rd, Charlottesville, VA 22903, USA \\ e-mail: thunter@nrao.edu \\ 5 European Southern Observatory, Karl-Schwarzschild-Str. 2, 85748 Garching, Germany \\ e-mail: sleurini@eso.org
}

Received 18 December 2006 / Accepted 3 April 2007

\section{ABSTRACT}

\begin{abstract}
Context. Hot molecular cores are an early manifestation of massive star formation where the molecular gas is heated to temperatures $>100 \mathrm{~K}$ undergoing a complex chemistry.

Aims. One wants to better understand the physical and chemical processes in this early evolutionary stage.

Methods. We selected the prototypical hot molecular core G29.96-0.02 being located at the head of the associated ultracompact HII region. The $862 \mu \mathrm{m}$ submm continuum and spectral line data were obtained with the Submillimeter Array (SMA) at sub-arcsecond spatial resolution.

Results. The SMA resolved the hot molecular core into six submm continuum sources with the finest spatial resolution of $0.36^{\prime \prime} \times 0.25^{\prime \prime}$ $(\sim 1800 \mathrm{AU})$ achieved so far. Four of them located within $7800(\mathrm{AU})^{2}$ comprise a proto-Trapezium system with estimated protostellar densities of $1.4 \times 10^{5}$ protostars $/ \mathrm{pc}^{3}$. The plethora of $\sim 80$ spectral lines allows us to study the molecular outflow(s), the core kinematics, the temperature structure of the region as well as chemical effects. The derived hot core temperatures are of the order $300 \mathrm{~K}$. We find interesting chemical spatial differentiations, e.g., $\mathrm{C}^{34} \mathrm{~S}$ is deficient toward the hot core and is enhanced at the UCHII/ hot core interface, which may be explained by temperature sensitive desorption from grains and following gas phase chemistry. The $\mathrm{SiO}(8-7) \mathrm{emission}$ outlines likely two molecular outflows emanating from this hot core region. Emission from most other molecules peaks centrally on the hot core and is not dominated by any individual submm peak. Potential reasons for that are discussed. A few spectral lines that are associated with the main submm continuum source, show a velocity gradient perpendicular to the large-scale outflow. Since this velocity structure comprises three of the central protostellar sources, this is not a Keplerian disk. While the data are consistent with a gas core that may rotate and/or collapse, we cannot exclude the outflow(s) and/or nearby expanding UCHII region as possible alternative causes of this velocity pattern
\end{abstract}

Key words. stars: formation - ISM: jets and outflows - ISM: molecules - stars: early-type - stars: individual: G29.96-0.02 stars: binaries: close

\section{Introduction}

Hot molecular cores represent an early evolutionary stage in massive star formation prior to the formation of an ultracompact HII region (UCHII). Single-dish line surveys toward hot cores have revealed high abundances of many molecular species and temperatures usually exceeding 100 K (e.g., Schilke et al. 1997; Hatchell et al. 1998; McCutcheon et al. 2000). Unfortunately, most hot cores are relatively far away (a few kpc, Orion-KL being an important exception), and high-spatial-resolution studies are important to disentangle the various components in the region, to resolve potential multiple heating sources, and to search for chemical variations throughout the regions. Here we present sub-arcsecond resolution submm spectral line and dust continuum observations of the hot core G29.96-0.02, characterizing the physical and chemical properties of this prototypical region.

The hot core/UCHII region G29.96-0.02 is a well studied source comprising a cometary UCHII region and approximately
2.6" to the west a hot molecular core (Wood \& Churchwell 1989; Cesaroni et al. 1994, 1998). G29.96-0.02 is at a distance of $\sim 6 \mathrm{kpc}$ (Pratap et al. 1999), the bolometric luminosity measured with IRAS is very high with $L \sim 1.4 \times 10^{6} L_{\odot}$ (Cesaroni et al. 1994). Since the region harbors at least two massive (proto)stars (within the UCHII region and the hot core) this luminosity must be distributed over various sources. Based on $\mathrm{cm}$ continuum free-free emission, Cesaroni et al. (1994) calculate a luminosity for the UCHII region of $L_{\mathrm{cm}} \sim 4.4 \times 10^{5} L_{\odot}$. Furthermore, they try to estimate the luminosity of the hot core via a first order blackbody approximation and get a value of $L_{\mathrm{bb}} \sim 1.2 \times 10^{5} L_{\odot}$. Later, Olmi et al. (2003) derive a similar estimate $\left(\sim 9 \times 10^{4} L_{\odot}\right)$ via integrating a much better determined SED. The exciting source of the UCHII region has been identified in the near-infrared as an O5-O8 star (Watson \& Hanson 1997). Furthermore, Pratap et al. (1999) identified two additional sources toward the rim of the UCHII region and an enhanced density of reddened sources indicative of an embedded cluster. 
A line survey toward a number of UCHII regions reveals that G29.96-0.02 is a strong molecular line emitter in nearly all observed species (Hatchell et al. 1998). High-angular resolution studies show that many species (e.g., $\mathrm{NH}_{3}, \mathrm{CH}_{3} \mathrm{CN}$, $\mathrm{HNCO}, \mathrm{HCOOCH}_{3}$ ) peak toward the main $\mathrm{H}_{2} \mathrm{O}$ maser cluster $\sim 2.6^{\prime \prime}$ west of the UCHII region (e.g, Hofner \& Churchwell 1996; Cesaroni et al. 1998; Olmi et al. 2003), whereas $\mathrm{CH}_{3} \mathrm{OH}$ peaks $\sim 4$ " further south-west associated with another isolated $\mathrm{H}_{2} \mathrm{O}$ maser feature (Pratap et al. 1999). Hoffman et al. (2003) detected one of the relatively rare $\mathrm{H}_{2} \mathrm{CO}$ masers toward the hot core position. These masers are proposed to trace the warm molecular gas in the vicinity of young forming massive stars (Araya et al. 2006). The signature of a $\mathrm{CH}_{3} \mathrm{OH}$ peak offset from the other molecular lines is reminiscent of Orion-KL (e.g., Wright et al. 1996; Beuther et al. 2005b). Temperature estimates toward the hot core based on high-density tracers vary between 80 and 150 K (e.g., Cesaroni et al. 1994; Hatchell et al. 1998; Pratap et al. 1999; Olmi et al. 2003).

While Gibb et al. (2004) detect a molecular outflow in $\mathrm{H}_{2} \mathrm{~S}$ emanating from the hot core in approximately the south-east north-west direction, Cesaroni et al. (1998) and Olmi et al. (2003) detect a velocity gradient in the east-west direction in the high-density tracers $\mathrm{NH}_{3}(4,4)$ and $\mathrm{CH}_{3} \mathrm{CN}$, consistent with a rotating disk around an embedded protostar. However, Maxia et al. (2001) also report that their rather low-resolution 5.9" $\times 3.7^{\prime \prime}$ $(\approx 0.15 \mathrm{pc}) \mathrm{SiO}(2-1)$ data are consistent with the disk scenario as well. This is a bit puzzling since $\mathrm{SiO}$ is usually found to trace shocked gas in outflows and not more quiescent gas in disks. Inspecting their SiO image again (Fig. 6 in Maxia et al. 2001), this interpretation is not unambiguous, the data also appear to be consistent with the outflow observed in $\mathrm{H}_{2} \mathrm{~S}$ (Gibb et al. 2004). It is possible that the spatial resolution of their $\mathrm{SiO}(2-1)$ observations is not sufficient to really disentangle the outflow in this distant region.

Olmi et al. (2003) compiled the SED from cm to midinfrared wavelengths. While the $3 \mathrm{~mm}$ data are still strongly dominated by the free-free emission (Olmi et al. 2003), at $1 \mathrm{~mm}$ the hot core becomes clearly distinguished from the adjacent UCHII region (Wyrowski et al. 2002). G29.96-0.02 is one of the few hot cores which is detected at mid-infrared wavelengths (De Buizer et al. 2002). Interestingly, the mid-infrared peak is $\sim 0.5^{\prime \prime}(\sim 3000 \mathrm{AU})$ offset from the $\mathrm{NH}_{3}(4,4)$ hot core position. While Gibb et al. (2004) speculate that the mid-infrared peak might arise from the scattered light only, De Buizer et al. (2002) suggest that it could trace a second massive source within the same core. This hypothesis can be tested via very-high-angularresolution submm continuum studies.

\section{Observations}

We have observed the hot core G29.96-0.02 with the Submillimeter Array (SMA ${ }^{1}$, Ho et al. 2004) during four nights between May and November 2005. We used all available array configurations (compact, extended, very extended, for details see Table 1) with unprojected baselines between 16 and $500 \mathrm{~m}$, resulting at $862 \mu \mathrm{m}$ in a projected baseline range from 16.5 to $591 \mathrm{k} \lambda$. The chosen phase center was the peak position of the associated UCHII region RA [J2000.0]: $18^{\mathrm{h}} 46^{\mathrm{m}} 03^{\mathrm{s}} .99$ and

1 The Submillimeter Array is a joint project between the Smithsonian Astrophysical Observatory and the Academia Sinica Institute of Astronomy and Astrophysics, and is funded by the Smithsonian Institution and the Academia Sinica.
Table 1. Observing parameters.

\begin{tabular}{lrrrr}
\hline \hline Date & Config. & \# ant. & $\begin{array}{r}\text { Source loop } \\
\text { [hours] }\end{array}$ & $\tau(225 \mathrm{GHz})$ \\
\hline 28 May 05 & very ext. & 6 & 7.0 & $0.13-0.16$ \\
18 Jul. 05 & comp. & 7 & 7.5 & $0.06-0.09$ \\
4 Sep. 05 & ext. & 6 & 4.5 & $0.06-0.08$ \\
5 Nov. 05 & very ext. & 7 & 3.0 & 0.06 \\
\hline
\end{tabular}

Dec [J2000.0] $-02^{\circ} 39^{\prime} 21^{\prime \prime} .47$. The velocity of rest is $v_{\mathrm{lsr}} \sim$ $+98 \mathrm{~km} \mathrm{~s}^{-1}$ (Churchwell et al. 1990).

For bandpass calibration we used Ganymede in the compact configuration and 3C 279 and 3C 454.3 in the extended and very extended configuration. The flux scale was derived in the compact configuration again from observations of Ganymede. For two datasets of the more extended configurations, we used 3C 454.3 for the relative scaling between the various baselines and then scaled that absolutely via observations of Uranus. For the fourth dataset we did the flux calibration using 3C 279 only. The flux accuracy is estimated to be accurate within $20 \%$. Phase and amplitude calibration was done via frequent observations of the quasars $1743-038$ and $1751+096$, about $15.5^{\circ}$ and $18.3^{\circ}$ from the phase center of G29.96-0.02. The zenith opacity $\tau(348 \mathrm{GHz})$, measured with the NRAO tipping radiometer located at the Caltech Submillimeter Observatory, varied during the different observation nights between $\sim 0.15$ and $\sim 0.4$ (scaled from the $225 \mathrm{GHz}$ measurement). The receiver operated in a double-sideband mode with an IF band of $4-6 \mathrm{GHz}$ so that the upper and lower sideband were separated by $10 \mathrm{GHz}$. The central frequencies of the upper and lower sideband were 348.2 and $338.2 \mathrm{GHz}$, respectively. The correlator had a bandwidth of $2 \mathrm{GHz}$ and the channel spacing was $0.8125 \mathrm{MHz}$. Measured double-sideband system temperatures corrected to the top of the atmosphere were between 110 and $800 \mathrm{~K}$, depending on the zenith opacity and the elevation of the source. Our sensitivity was dynamic-range limited by the side-lobes of the strongest emission peaks and thus varied between the line maps of different molecules and molecular transitions. This limitation was mainly due to the incomplete sampling of short uv-spacings and the presence of extended structures. The $1 \sigma$ rms for the velocityintegrated molecular line maps (the velocity ranges for the integrations were chosen for each line separately depending on the line-widths and intensities) ranged between 36 and $76 \mathrm{mJy}$. The average synthesized beam of the spectral line maps was $0.65^{\prime \prime} \times 0.48^{\prime \prime}\left(\mathrm{PA}-83^{\circ}\right)$. The $862 \mu \mathrm{m}$ submm continuum image was created by averaging the apparently line-free parts of the upper sideband. The $1 \sigma$ rms of the submm continuum image was $\sim 21 \mathrm{mJy} /$ beam, and the achieved synthesized beam was $0.36^{\prime \prime} \times 0.25^{\prime \prime}\left(\mathrm{PA} 18^{\circ}\right)$, the smallest beam obtained so far with the SMA. The different synthesized beams between line and continuum maps are due to different applied weightings in the imaging process ("robust" parameters set in MIRIAD to 0 and -2, respectively) because there was insufficient signal-to-noise in the line data obtained in the very extended configuration. The initial flagging and calibration was done with the IDL superset MIR originally developed for the Owens Valley Radio Observatory (Scoville et al. 1993) and adapted for the SMA ${ }^{2}$. The imaging and data analysis were conducted in MIRIAD (Sault et al. 1995).

\footnotetext{
2 The MIR cookbook by Charlie Qi can be found at http://cfa-www . harvard. edu/ cqi/mircook . html
} 


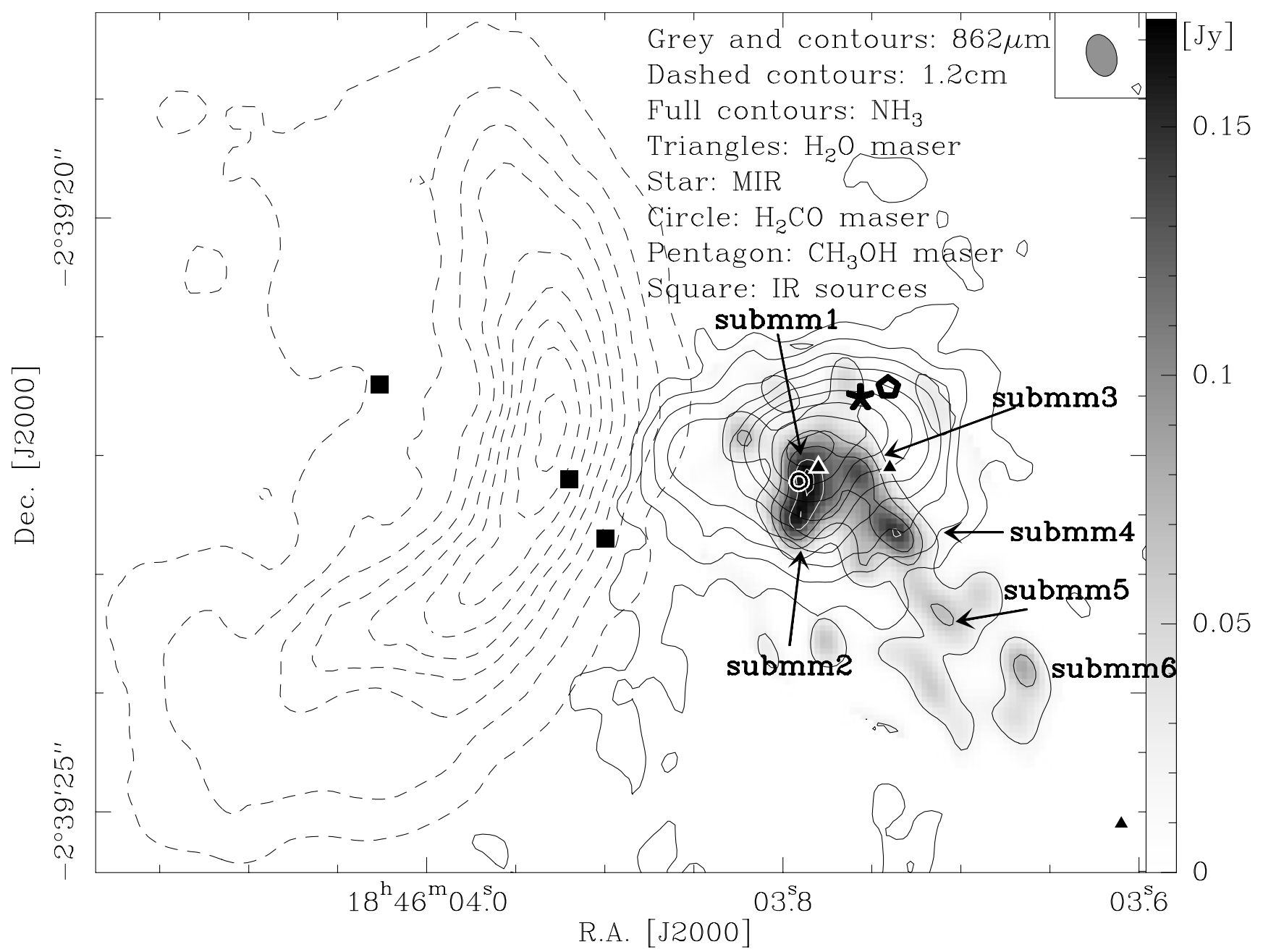

Fig. 1. The hot core UCHII region G29.96-0.02. The grey-scale with contours shows the submm continuum emission with a spatial resolution of $0.36^{\prime \prime} \times 0.25^{\prime \prime}$. The contour levels start at the $1 \sigma$ level of $21 \mathrm{mJy}$ beam ${ }^{-1}$ and continue at $63,105 \mathrm{mJy}^{-1}$ beam ${ }^{-1}$ (black contours) to 147 , $168 \mathrm{mJy}$ beam $^{-1}$ (white contours). The dashed contours outline the $\mathrm{cm}$ continuum emission from the UCHII region and the thick contours show the $\mathrm{NH}_{3}$ emission (Cesaroni et al. 1994). The contouring is done from 15 to $95 \%$ (step 10\%) of the peak emission of each image, respectively $\left(S_{\text {peak }}(1.2 \mathrm{~cm})=109 \mathrm{mJy} /\right.$ beam, $S_{\text {peak }}\left(\mathrm{NH}_{3}\right)=15 \mathrm{mJy} /$ beam $)$. Triangles, circles and pentagons show the $\mathrm{H}_{2} \mathrm{O}$ (Hofner \& Churchwell 1996), $\mathrm{H}_{2} \mathrm{CO}$ (Hoffman et al. 2003) and class II $\mathrm{CH}_{3} \mathrm{OH}$ (Minier et al. 2001) maser positions. The star marks the peak of the MIR emission (De Buizer et al. 2002), which is not a point source but has a similar size as the $\mathrm{NH}_{3}$ emission. The squares mark the infrared sources by Pratap et al. (1999).

\section{Results}

\subsection{Submillimeter continuum emission}

Figure 1 presents the $862 \mu \mathrm{m}$ continuum emission extracted from the line-free parts of the upper sideband spectrum $(\sim 1.8 \mathrm{GHz}$ in total used) shown in Fig. 3. The very high spatial resolution of $0.36^{\prime \prime} \times 0.25^{\prime \prime}$ corresponds to a linear resolution of $\sim 1800 \mathrm{AU}$ at the given distance of $\sim 6 \mathrm{kpc}$. The submm continuum emission peaks approximately $2^{\prime \prime}$ west of the UCHII region and is associated with the molecular line emission known from previous observations. We do not detect any submm continuum emission toward the UCHII region itself. At the given spatial resolution, for the first time multiplicity within the G29.96-0.02 hot core is resolved and we identify 6 submm continuum emission peaks (submm1 to submm6) above the $3 \sigma$ level of $63 \mathrm{mJy} \mathrm{beam}^{-1}$ (Fig. 1). We consider submm1 and submm2 to be separate sources instead of a dust ridge because we count compact spherical or elliptical sources and their emission peaks are separated by about one synthesized beam. The four strongest submm peaks, that are all $>6 \sigma$ detections, are located within a region of $\left(1.3^{\prime \prime}\right)^{2}$ (7800 AU) in diameter. The submm peak submm1 is associated with $\mathrm{H}_{2} \mathrm{O}$ and $\mathrm{H}_{2} \mathrm{CO}$ maser emission (Hofner \& Churchwell 1996; Hoffman et al. 2003), and we consider this to be probably the most luminous sub-source. The other $\mathrm{H}_{2} \mathrm{O}$ maser peaks are offset from the submm continuum emission. The mid-infrared source detected by De Buizer et al. (2002) is offset $>1^{\prime \prime}$ from the submm emission. This may either be due to uncertainties in the MIR astrometry or the MIR emission may trace another young source in the region. It should be noted that the class II $\mathrm{CH}_{3} \mathrm{OH}$ masers detected by Minier et al. (2001) peak close to the MIR source as well, which indicates that the MIR offset from the hot core may well be real.

Table 2 lists the absolute source positions, their $862 \mu \mathrm{m}$ peak intensities and the integrated flux densities approximately associated with each of the sub-sources. Calculating the brightness temperature $T_{\mathrm{b}}$ of the corresponding Planck-function for, e.g., submm1, we get $T_{\mathrm{b}}(\mathrm{Peak} 1) \sim 27 \mathrm{~K}$. Assuming hot core dust temperatures of $\sim 100 \mathrm{~K}$, the usual assumption of optically thin dust emission is not really valid anymore, and one gets an approximate beam-averaged optical depth $\tau$ of the dust emission of $\sim 0.3$. To calculate the dust and gas masses, we can follow the mass determination outlined in Hildebrand (1983) and 
Table 2. Submm continuum source parameters.

\begin{tabular}{lrrrrrr}
\hline \hline Source & $\begin{array}{r}\text { RA } \\
{[\mathrm{J} 2000]}\end{array}$ & $\begin{array}{r}\text { Dec } \\
{[\mathrm{J} 2000]}\end{array}$ & $\begin{array}{r}S_{\text {peak }} \\
{\left[\frac{\mathrm{mJy}}{\text { beam }}\right]}\end{array}$ & $\begin{array}{r}S_{\text {int }} \\
{[\mathrm{mJy}]}\end{array}$ & $\begin{array}{r}M \\
{\left[M_{\odot}\right]}\end{array}$ & $\begin{array}{r}N \\
{\left[10^{24} \mathrm{~cm}^{-3}\right]}\end{array}$ \\
\hline submm1 & $18: 46: 03.786$ & $-02: 39: 22.19$ & 173 & 288 & 11.5 & 5.7 \\
submm2 & $18: 46: 03.789$ & $-02: 39: 22.48$ & 168 & 237 & 9.5 & 5.5 \\
submm3 & $18: 46: 03.758$ & $-02: 39: 22.16$ & 138 & 178 & 7.1 & 4.5 \\
submm4 & $18: 46: 03.736$ & $-02: 39: 22.65$ & 151 & 249 & 9.9 & 5.0 \\
submm5 & $18: 46: 03.710$ & $-02: 39: 23.33$ & 68 & 106 & 4.2 & 2.2 \\
submm6 & $18: 46: 03.665$ & $-02: 39: 23.80$ & 84 & 85 & 3.4 & 2.8 \\
\hline
\end{tabular}

The table shows the peak intensities $S_{\text {peak }}$, the integrated intensities $S_{\text {int }}$, the derived gas masses $M$ as well as the $\mathrm{H}_{2}$ column densities $N$.

Beuther et al. (2002, 2005a), which assumes optically thin emission, and correct that for the increased dust opacity. Assuming constant emission along the line of sight, the opacity correction factor $C$ is

$C=\frac{\tau}{1-\mathrm{e}^{-\tau}}$

With $\tau \sim 0.3$, we get a correction factor $C \sim 1.16$ still comparably small. Assuming a dust opacity index $\beta=1.5$, the dust opacity per unit dust mass is $\kappa(862 \mu \mathrm{m}) \sim 1.5 \mathrm{~cm}^{2} \mathrm{~g}^{-1}$ (with the reference value $\kappa(250 \mu \mathrm{m}) \sim 9.4 \mathrm{~cm}^{2} \mathrm{~g}^{-1}$, see Hildebrand 1983), and we assume a gas-to-dust ratio of 100 . Given the uncertainties in $\beta$ and $T$, we estimate the masses to be accurate within a factor 4 . Table 2 gives the derived masses and beam-averaged column densities. Each sub-peak has a mass of a few $M_{\odot}$, and the main submm 1 exhibits approximately $10 M_{\odot}$ of compact, warm gas and dust emission. The integrated $862 \mu \mathrm{m}$ continuum flux density of the central region comprising the four main submm continuum sources amounts to $1.16 \mathrm{Jy}$. At an average dust temperature of $100 \mathrm{~K}$, this corresponds to a central core mass of $39.9 M_{\odot}$. In comparison to these flux density measurements, Thompson et al. (2006) observed with SCUBA $850 \mu \mathrm{m}$ peak and integrated flux densities of $\sim 11.5 \pm 1.2 \mathrm{Jy} /\left(14^{\prime \prime}\right.$ beam $)$ and $\sim 19.2 \mathrm{Jy}$, respectively. The ratio between peak and integrated JCMT fluxes already indicates non-compact emission even on that scales. Furthermore, subtracting a typical line contamination of the continuum emission in hot cores of the order $25 \%$ (e.g., NGC 6334I, Hunter et al. in prep.), the total $850 \mu \mathrm{m}$ single-dish continuum flux density should amount to $\sim 8.6 \mathrm{Jy}$. Compared with the integrated flux density in the SMA data of $\sim 1.74 \mathrm{Jy}$, this indicates that approximately $80 \%$ of the single-dish emission is filtered out by the missing short spacings in the interferometer data. The dust and gas in the central region have higher temperatures than the components filtered out on larger spatial scales, and since the dust and gas mass is inversely proportionally related to the temperature by $M_{\mathrm{H}_{2}} \propto\left(\mathrm{e}^{h v / k T}-1\right)$ (e.g., Beuther et al. $2002)$, a greater proportion of the mass $(>80 \%)$ is filtered out in the SMA data. However, the SMA image reveals the most compact hot gas and dust cores at the center of the evolving massive star-forming region. The shortest baseline of the SMA observations of $\sim 16.5 \mathrm{k} \lambda$ correspond to scales $>12^{\prime \prime}$ which hence have to be filtered out entirely. However, even smaller scales are missing because the uv-spacings corresponding to scales $\geq 5^{\prime \prime}$ are still relatively poorly sampled and the image presented in Fig. 1 is only sensitive to spatial scales of the order a few arcseconds. The submm peaks detected by the SMA are much stronger than what would have been expected if the single-dish flux $(\sim 8.6 \mathrm{Jy})$ were uniformly distributed over the SCUBA primary beam of $14^{\prime \prime}$, even ignoring any spatial filtering and missing flux effects (This would result in $\sim 4$ mJy per synthesized SMA beam.). This shows that the emission measured on the small spatial scales sampled by the SMA represents the compact core emission much better than expected. However, it does not imply that the gas masses measured by the SMA are the only gas reservoir the embedded protostars have for their ongoing accretion; they may also gain mass from the large-scale gas envelope that is filtered out by our observations (see also the competitive accretion scenario, e.g., Bonnell et al. 2004). The derived beam-averaged $\mathrm{H}_{2}$ column densities are of the order a few times $10^{24} \mathrm{~cm}^{-2}$, corresponding to visual extinctions $A_{\mathrm{v}}$ of a few $1000\left(A_{\mathrm{v}}=N_{\mathrm{H}} / 0.94 \times 10^{21}\right.$, Frerking et al. 1982).

\subsection{Spectral line emission}

Figure 3 presents spectra extracted toward the main submm submm1 with an angular resolution of $0.64^{\prime \prime} \times 0.47^{\prime \prime}$ compared to the submm continuum map (see Sect. 2). More than 80 spectral lines from 18 molecular species, isotopologues or vibrationally excited species have been identified with a minor fraction of $\sim 5 \%$ of unidentified lines (UL) (Tables 6 and 4). The range of upper level excitation temperatures for the many lines varies between approximately 40 and $750 \mathrm{~K}$ (Table 6). Therefore, with one set of observations we are able to trace various gaseous temperature components from the relatively colder gas surrounding the hot core region to the densest and warmest gas best observed in some of the vibrationally excited lines.

Figure 2 now presents integrated images of the various detected species, isotopologues and vibrationally excited lines. For comparison, Fig. 2 also shows the submm continuum emission reduced with the same degraded spatial resolution as the line images. All images show emission in the vicinity of the hot molecular core and no emission toward the associated UCHII region. However, the morphology varies significantly between many of the observed molecular line maps. The molecular emission is largely confined to the central region of the main four submm continuum peaks, and we do not detect appreciable molecular emission toward the continuum peaks 5 and 6 . Reducing the submm continuum data with the same spatial resolution as the line images, the four submm peaks are smoothed to a single elongated structure peaking close to the submm peak submm1 (Fig. 2, top-left panel). The ground state $\mathrm{CH}_{3} \mathrm{OH}$ emission is relatively broadly distributed with two peaks in east-west direction, and one may associate one with the submm peaks 1 and 2 and the other with the submm peak submm3, but most other maps show on average one spectral line peak somewhere in the middle of the 4 main submm continuum peaks, similar to the lower-resolution submm continuum map.

However, there are also a few species which significantly deviate from this picture and show a different spatial morphology. For example $\mathrm{SiO}$ is more extended in north-east south-west direction likely due to a molecular outflow (Sect. 3.3). Also interesting is the emission from $\mathrm{C}^{34} \mathrm{~S}$ which lacks emission around the central four submm peaks but is stronger in the interface 


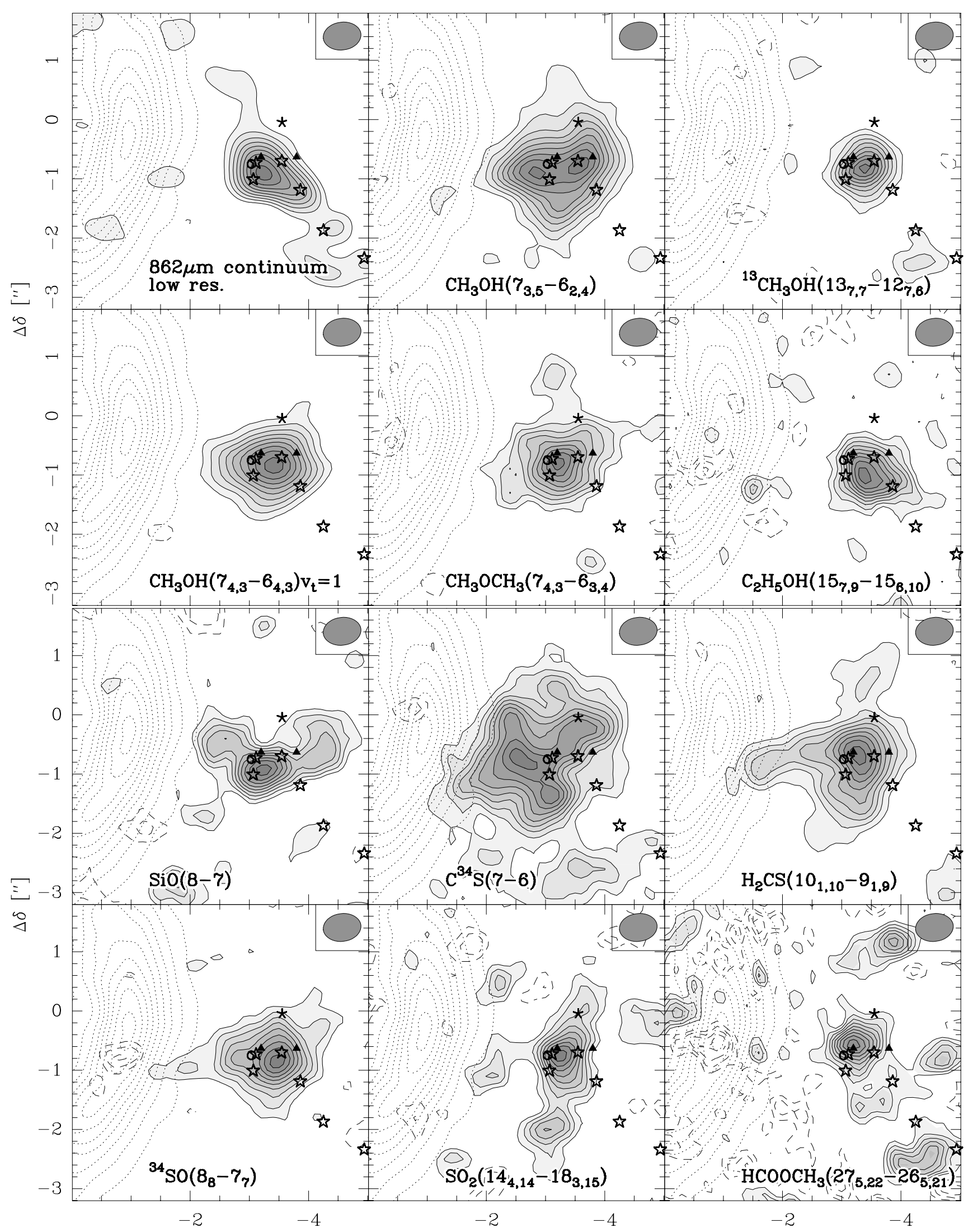

Fig. 2. Compilation of integrated line images (and submm continuum at the same spatial resolution) always shown in grey-scale with contours and labeled at the bottom of each panel. The dashed contours show negative features due to missing short spacings. The contouring is done from \pm 15 to $\pm 95 \%$ (step $\pm 10 \%$ ) of the peak emission of each image, respectively. Peak fluxes $S_{\text {peak }}$, rms and integrated velocity ranges for each image are given in Table 3. The dotted contours again show the UCHII region and the stars mark the submm continuum peaks from Fig. 1. The offsets on the axes are relative to the phase center. 


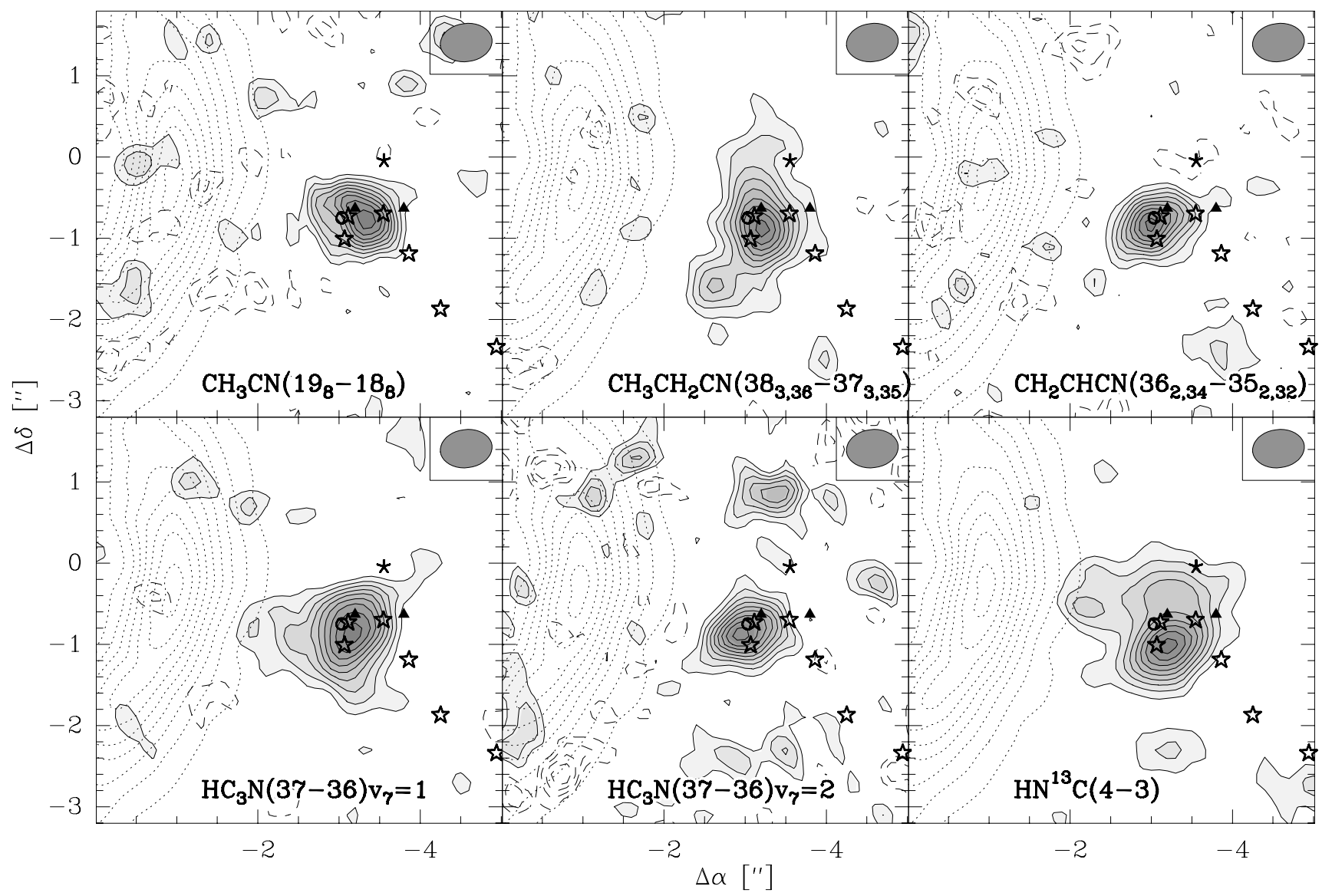

Fig. 2. continued.

region between the hot molecular core and the UCHII region (Sect. 4.3). Furthermore, there are a few spectral line maps - mainly those from likely optically thin lines $\left(\mathrm{HCOOCH}_{3}\right.$, $\left.\mathrm{HN}^{13} \mathrm{C}\right)$, highly excited lines $\left(\mathrm{CH}_{3} \mathrm{CHCN}\right)$ and vibrationally excited lines $\left(\mathrm{CH}_{3} \mathrm{OH} v_{\mathrm{t}}=1,2, \mathrm{HC}_{3} \mathrm{~N} v_{7}=1,2\right)$ - which show their emission peaks concentrated toward the main submm peak submm1 (Sect. 4.5).

Previous lower-resolution $\left(\sim 10^{\prime \prime}\right)$ molecular line observations revealed strong $\mathrm{CH}_{3} \mathrm{OH}$ emission toward the $\mathrm{H}_{2} \mathrm{O}$ maser feature approximately $4^{\prime \prime}$ south-west of the hot core peak (Fig. 1, Hofner \& Churchwell 1996; Pratap et al. 1999). A little bit surprising, we do not detect any $\mathrm{CH}_{3} \mathrm{OH}$ emission (nor any other species) toward that south-western position, even when imaged at low angular resolution using only the compact configuration data (therefore, we do not cover that position in Fig. 2). Pratap et al. (1999) discuss mainly two possibilities to explain this discrepancy: Either their observed specific $\mathrm{CH}_{3} \mathrm{OH}\left(8_{0}-7_{1}\right)$ line is a weak maser and we do not cover any comparable $\mathrm{CH}_{3} \mathrm{OH}$ line, or the emission covered by the lower-resolution data is relatively extended and filtered out by our observations. As discussed in the previous section, the shortest baseline of our observations was $\sim 16 \mathrm{~m}$, implying that we are not sensitive to any scales $>12^{\prime \prime}$. Since the $\mathrm{CH}_{3} \mathrm{OH}$ emission in Pratap et al. (1999) is slightly resolved by their synthesized beam of $12.6^{\prime \prime} \times 9.8^{\prime \prime}$, it is unlikely that we would have filtered out all emission. However, among the many observed $\mathrm{CH}_{3} \mathrm{OH}$ lines (Table 6), some have similar excitation temperatures of the order $80 \mathrm{~K}$ as the line observed by Pratap et al. (1999), and we would expect to detect thermal emission from these lines as well. Therefore, our non-detection of $\mathrm{CH}_{3} \mathrm{OH}$ emission toward the south-western
Table 3. Peak intensities, rms and velocity ranges for images in Fig. 2.

\begin{tabular}{lrrr}
\hline \hline Line & $\begin{array}{r}S_{\text {peak }} \\
\text { mJy/beam }\end{array}$ & $\begin{array}{r}\text { rms } \\
\text { mJy/beam }\end{array}$ & $\begin{array}{r}\Delta v \\
\mathrm{~km} \mathrm{~s}^{-1}\end{array}$ \\
\hline $862 \mu$ m cont., low res. & 422 & 17 & \\
$\mathrm{CH}_{3} \mathrm{OH}\left(7_{3,5}-6_{2,4}\right)$ & 878 & 64 & {$[90,104]$} \\
${ }^{13} \mathrm{CH}_{3} \mathrm{OH}\left(13_{7,7}-12_{7,6}\right)$ & 752 & 51 & {$[95,101]$} \\
$\mathrm{CH}_{3} \mathrm{OH}\left(7_{4,3}-6_{4,3}\right), v_{\mathrm{t}}=1$ & 1419 & 69 & {$[91,105]$} \\
$\mathrm{CH}_{3} \mathrm{OCH}{ }_{3}\left(7_{4,3}-6_{3,4}\right)$ & 669 & 46 & {$[94,104]$} \\
$\mathrm{C}_{2} \mathrm{H}_{5} \mathrm{OH}\left(15_{7,9}-15_{6,10}\right)$ & 586 & 51 & {$[95,100]$} \\
$\mathrm{SiO}(8-7)$ & 391 & 36 & {$[75,105]$} \\
$\mathrm{C}^{34} \mathrm{~S}(7-6)$ & 592 & 62 & {$[92,104]$} \\
$\mathrm{H}_{2} \mathrm{CS}\left(10_{1,0}-9_{1,9}\right)$ & 933 & 69 & {$[92,100]$} \\
${ }^{34} \mathrm{SO}\left(8_{8}-7_{7}\right)$ & 827 & 57 & {$[95,103]$} \\
$\mathrm{SO}_{2}\left(14_{4,14}-18_{3,15}\right)$ & 544 & 53 & {$[94,100]$} \\
$\mathrm{HCOOCH}_{3}\left(27_{5,22}-26_{5,21}\right)$ & 491 & 70 & {$[96,100]$} \\
$\mathrm{CH}_{3} \mathrm{CN}\left(19_{8}-18_{8}\right)$ & 788 & 71 & {$[94,100]$} \\
$\mathrm{CH}_{3} \mathrm{CH} \mathrm{H}_{2} \mathrm{CN}\left(38_{3,36}-37_{3,35}\right)$ & 791 & 56 & {$[94,102]$} \\
$\mathrm{CH}_{3} \mathrm{CHCN}\left(36_{2,34}-35_{2,32}\right)$ & 655 & 68 & {$[96,100]$} \\
$\mathrm{HC}_{3} \mathrm{~N}(37-36), v_{7}=1$ & 622 & 55 & {$[94,102]$} \\
$\mathrm{HC}_{3} \mathrm{~N}(37-36), v_{7}=2$ & 416 & 57 & {$[94,100]$} \\
$\mathrm{HN}^{13} \mathrm{C}(4-3)$ & 1149 & 76 & {$[94,100]$} \\
\hline
\end{tabular}

$\mathrm{H}_{2} \mathrm{O}$ maser position supports rather their suggested scenario of weak $\mathrm{CH}_{3} \mathrm{OH}$ maser emission in the previously reported observations (Pratap et al. 1999).

\subsection{Molecular outflow emission}

The $\mathrm{SiO}(8-7)$ spectrum spans a large range of velocities from $\sim 75$ to $\sim 111 \mathrm{~km} \mathrm{~s}^{-1}$. Integrating the blue- and red-shifted 

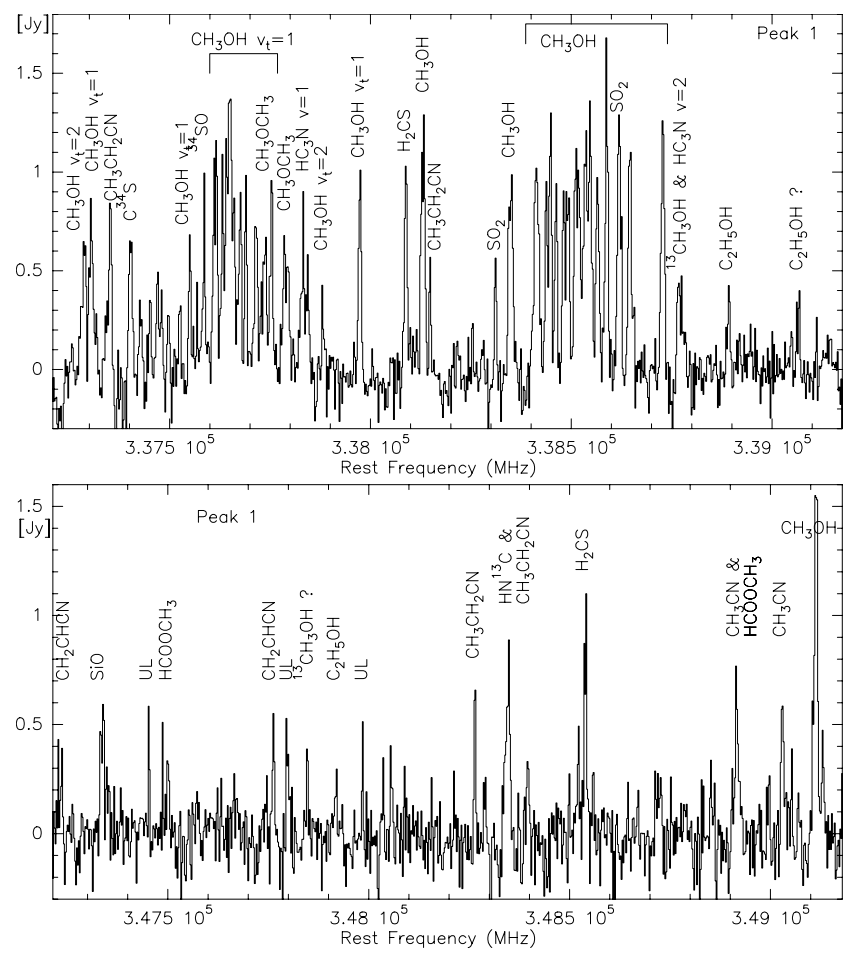

Fig. 3. Lower and upper sideband spectra extracted toward the submm1. The spatial resolution of these data is $0.64^{\prime \prime} \times 0.47^{\prime \prime}$. The main line identifications are shown in both panels.

Table 4. Detected molecular species.

\begin{tabular}{|c|c|c|}
\hline Species & Isotopologues & Vibrational states \\
\hline $\begin{array}{l}\mathrm{CH}_{3} \mathrm{OH} \\
\mathrm{CH}_{3} \mathrm{OCH}_{3} \\
\mathrm{C}_{2} \mathrm{H}_{5} \mathrm{OH} \\
\mathrm{SiO}\end{array}$ & ${ }^{13} \mathrm{CH}_{3} \mathrm{OH}$ & $\mathrm{CH}_{3} \mathrm{OH}, v_{\mathrm{t}}=1,2^{a}$ \\
\hline $\mathrm{H}_{2} \mathrm{CS}$ & $\mathrm{C}^{34} \mathrm{~S}$ & \\
\hline $\begin{array}{l}\mathrm{SO}_{2} \\
\mathrm{HCOOCH}_{3} \\
\mathrm{CH}_{3} \mathrm{CN} \\
\mathrm{CH}_{3} \mathrm{CH}_{2} \mathrm{CN} \\
\mathrm{CH}_{3} \mathrm{CHCN}\end{array}$ & ${ }^{34} \mathrm{SO}$ & \\
\hline & $\mathrm{HN}^{13} \mathrm{C}$ & $\mathrm{HC}_{3} \mathrm{~N}, v_{7}=1,2$ \\
\hline
\end{tabular}

${ }^{a}$ The detection of this $\mathrm{CH}_{3} \mathrm{OH} v_{\mathrm{t}}=2$ line is doubtful since other close $v_{\mathrm{t}}=2$ lines with similar excitation temperatures were not detected.

emission, one gets the outflow map presented in Fig. 4. The elongated north-west south-east structure is consistent with the previously proposed outflow by Gibb et al. (2004). The additional red feature north-east of the central hot core region makes the interpretation ambiguous: if the north-west south-east outflow is a relatively highly collimated jet, then the north-eastern red feature could be attributed to an additional outflow leaving the core in north-east south-west direction. The blue wing of that potential second outflow would not detected in our data. However, since we are filtering out any larger-scale emission, it is also possible that the red $\mathrm{SiO}$ features south-east and north-east of the main core are part of the same wide-angle outflow tracing potentially the limb-brightened cavity walls. In this scenario, our observations would miss part of the blue-shifted wide-angle

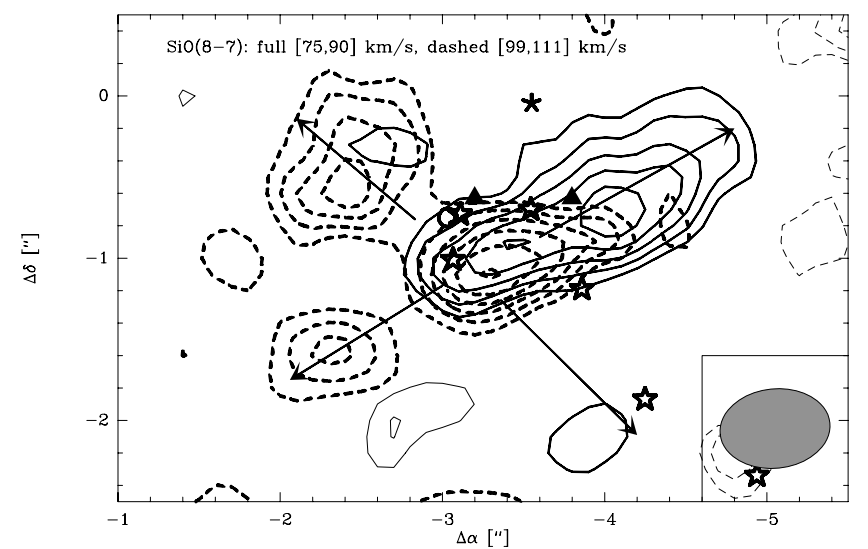

Fig. 4. $\mathrm{SiO}(8-7)$ outflow map. The full and dashed contours are integrated over the blue- and redshifted $\mathrm{SiO}$ emission as shown in the figure. The contouring starts at $\pm 2 \sigma$ and continues in $\pm 1 \sigma$ steps (thick contours positive, thin contours negative). The $1 \sigma$ values for the blue- and red-shifted images are 48 and $46 \mathrm{mJy}$ beam $^{-1}$, respectively. The markers are the same as in the previous images, the synthesized beam of $0.68^{\prime \prime} \times 0.49^{\prime \prime}$ is shown at the bottom right, and the arrows guide the eye for the potential directions of the two discussed outflows. The offsets on the axes are relative to the phase center.

Table 5. Spatial separation.

\begin{tabular}{ccr}
\hline \hline Pair & $\begin{array}{c}\Delta \theta \\
{\left[{ }^{\prime \prime}\right]}\end{array}$ & $\begin{array}{r}\Delta x \\
{[\mathrm{AU}]}\end{array}$ \\
\hline $1-2$ & 0.3 & 1800 \\
$1-3$ & 0.5 & 3000 \\
$1-4$ & 0.9 & 5400 \\
$2-3$ & 0.6 & 3600 \\
$2-4$ & 0.8 & 4800 \\
$3-4$ & 0.6 & 3600 \\
\hline
\end{tabular}

The numbers in Col. 1 correspond to the numbers of the submm peaks.

outflow lobe. With the current data, it is difficult to clearly distinguish between the two scenarios. However, comparing the elongated blue-shifted $\mathrm{SiO}(8-7)$ data with the previous north-west south-eastern outflow observed in $\mathrm{H}_{2} \mathrm{~S}$ by Gibb et al. (2004), it appears that this is the most likely direction of the main outflow of the region. Therefore, the multiple outflow scenario appears more likely for the hot core in G29.96-0.02. The lower resolution $\mathrm{SiO}(2-1)$ observation by Maxia et al. (2001) are also consistent with this scenario. Based on these data, we cannot conclusively say which of the submm continuum sources submm1 to submm 4 contribute to driving the outflows.

\section{Discussion}

\subsection{The formation of a proto-Trapezium system?}

The four main submm continuum peaks are located within a projected area of $7800 \times 7800(\mathrm{AU})^{2}$ on the sky. The projected separation $\Delta \theta$ between individual sub-sources varies between $1800 \mathrm{AU}$ (peaks 1 and 2) and $5400 \mathrm{AU}$ (peaks 1 and 4, see Table 5). Could the four central submm peaks be the predecessors of a future Trapezium system? Trapezia are defined as nonhierarchical multiple systems of three or more stars where the largest projected separation between Trapezia members should not exceed the smallest projected separation by a factor of 3 (Sharpless 1954; Ambartsumian 1955; Abt \& Corbally 2000). This criterion is satisfied by the four submm peaks at the center 
Table 6. Line parameters.

\begin{tabular}{|c|c|c|c|c|c|}
\hline $\begin{array}{l}\text { Freq. } \\
\text { GHz }\end{array}$ & Line & $\begin{array}{r}E_{u} \\
\mathrm{~K}\end{array}$ & $\begin{array}{r}\text { Freq. } \\
\text { GHz }\end{array}$ & Line & $\begin{array}{r}E_{u} \\
\mathrm{~K}\end{array}$ \\
\hline 337.279 & $\mathrm{CH}_{3} \mathrm{OH}\left(7_{2,5}-6_{2,4}\right) \mathrm{E}\left(v_{\mathrm{t}}=2\right)^{a}$ & 727 & 338.409 & $\mathrm{CH}_{3} \mathrm{OH}\left(7_{0,7}-6_{0,6}\right) \mathrm{A}$ & 65 \\
\hline 337.297 & $\mathrm{CH}_{3} \mathrm{OH}\left(7_{1,7}-6_{1,6}\right) \mathrm{A}\left(v_{\mathrm{t}}=1\right)$ & 390 & 338.431 & $\mathrm{CH}_{3} \mathrm{OH}\left(7_{6,1}-6_{6,0}\right) \mathrm{E}$ & 254 \\
\hline 337.348 & $\mathrm{CH}_{3} \mathrm{CH}_{2} \mathrm{CN}\left(38_{3,36}-37_{3,35}\right)$ & 328 & 338.442 & $\mathrm{CH}_{3} \mathrm{OH}\left(7_{6,1}-6_{6,0}\right) \mathrm{A}$ & 259 \\
\hline 337.397 & $C^{34} S(7-6)$ & 65 & & $\mathrm{CH}_{3} \mathrm{OH}\left(7_{6,2}-6_{6,1}\right) \mathrm{A}^{-}$ & 259 \\
\hline 337.421 & $\mathrm{CH}_{3} \mathrm{OCH}_{3}\left(21_{2,19}-20_{3,18}\right)$ & 220 & 338.457 & $\mathrm{CH}_{3} \mathrm{OH}\left(7_{5,2}-6_{5,1}\right) \mathrm{E}$ & 189 \\
\hline 337.446 & $\mathrm{CH}_{3} \mathrm{CH}_{2} \mathrm{CN}\left(37_{4,33}-36_{4,32}\right)$ & 322 & 338.475 & $\mathrm{CH}_{3} \mathrm{OH}\left(7_{5,3}-6_{5,2}\right) \mathrm{E}$ & 201 \\
\hline 337.464 & $\mathrm{CH}_{3} \mathrm{OH}\left(7_{6,1}-6_{0,0}\right) \mathrm{A}\left(v_{\mathrm{t}}=1\right)$ & 533 & 338.486 & $\mathrm{CH}_{3} \mathrm{OH}\left(7_{5,3}-6_{5,2}\right) \mathrm{A}$ & 203 \\
\hline 337.474 & UL & & & $\mathrm{CH}_{3} \mathrm{OH}\left(7_{5,2}-6_{5,1}\right) \mathrm{A}^{-}$ & 203 \\
\hline 337.490 & $\mathrm{HCOOCH}_{3}\left(27_{8,20}-26_{8,19}\right) \mathrm{E}$ & 267 & 338.504 & $\mathrm{CH}_{3} \mathrm{OH}\left(7_{4,4}-6_{4,3}\right) \mathrm{E}$ & 153 \\
\hline 337.519 & $\mathrm{CH}_{3} \mathrm{OH}\left(7_{5,2}-6_{5,2}\right) \mathrm{E}\left(v_{\mathrm{t}}=1\right)$ & 482 & 338.513 & $\mathrm{CH}_{3} \mathrm{OH}\left(7_{4,4}-6_{4,3}\right) \mathrm{A}^{-}$ & 145 \\
\hline \multirow[t]{2}{*}{337.546} & $\mathrm{CH}_{3} \mathrm{OH}\left(7_{5,3}-6_{5,2}\right) \mathrm{A}\left(v_{\mathrm{t}}=1\right)$ & 485 & & $\mathrm{CH}_{3} \mathrm{OH}\left(7_{4,3}-6_{4,2}\right) \mathrm{A}$ & 145 \\
\hline & $\mathrm{CH}_{3} \mathrm{OH}\left(7_{5,2}-6_{5,1}\right) \mathrm{A}^{-}\left(v_{\mathrm{t}}=1\right)$ & 485 & & $\mathrm{CH}_{3} \mathrm{OH}\left(7_{2,6}-6_{2,5}\right) \mathrm{A}^{-}$ & 103 \\
\hline 337.582 & ${ }^{34} \mathrm{SO}\left(8_{8}-7_{7}\right)$ & 86 & 338.530 & $\mathrm{CH}_{3} \mathrm{OH}\left(7_{4,3}-6_{4,2}\right) \mathrm{E}$ & 161 \\
\hline 337.605 & $\mathrm{CH}_{3} \mathrm{OH}\left(7_{2,5}-6_{2,4}\right) \mathrm{E}\left(v_{\mathrm{t}}=1\right)$ & 429 & 338.541 & $\mathrm{CH}_{3} \mathrm{OH}\left(7_{3,5}-6_{3,4}\right) \mathrm{A}^{+}$ & 115 \\
\hline \multirow[t]{2}{*}{337.611} & $\mathrm{CH}_{3} \mathrm{OH}\left(7_{6,1}-6_{6,0}\right) \mathrm{E}\left(v_{\mathrm{t}}=1\right)$ & 657 & 338.543 & $\mathrm{CH}_{3} \mathrm{OH}\left(7_{3,4}-6_{3,3}\right) \mathrm{A}^{-}$ & 115 \\
\hline & $\mathrm{CH}_{3} \mathrm{OH}\left(7_{3,4}-6_{3,3}\right) \mathrm{E}\left(v_{\mathrm{t}}=1\right)$ & 388 & 338.560 & $\mathrm{CH}_{3} \mathrm{OH}\left(7_{3,5}-6_{3,4}\right) \mathrm{E}$ & 128 \\
\hline 337.626 & $\mathrm{CH}_{3} \mathrm{OH}\left(7_{2,5}-6_{2,4}\right) \mathrm{A}\left(v_{\mathrm{t}}=1\right)$ & 364 & 338.583 & $\mathrm{CH}_{3} \mathrm{OH}\left(7_{3,4}-6_{3,3}\right) \mathrm{E}$ & 113 \\
\hline 337.636 & $\mathrm{CH}_{3} \mathrm{OH}\left(7_{2,6}-6_{2,5}\right) \mathrm{A}^{-}\left(v_{\mathrm{t}}=1\right)$ & 364 & 338.612 & $\mathrm{SO}_{2}\left(20_{1,19}-19_{2,18}\right)$ & 199 \\
\hline 337.642 & $\mathrm{CH}_{3} \mathrm{OH}\left(7_{1,7}-6_{1,6}\right) \mathrm{E}\left(v_{\mathrm{t}}=1\right)$ & 356 & 338.615 & $\mathrm{CH}_{3} \mathrm{OH}\left(7_{1,6}-6_{1,5}\right) \mathrm{E}$ & 86 \\
\hline 337.644 & $\mathrm{CH}_{3} \mathrm{OH}\left(7_{0,7}-6_{0,6}\right) \mathrm{E}\left(v_{\mathrm{t}}=1\right)$ & 365 & 338.640 & $\mathrm{CH}_{3} \mathrm{OH}\left(7_{2,5}-6_{2,4}\right) \mathrm{A}$ & 103 \\
\hline 337.646 & $\mathrm{CH}_{3} \mathrm{OH}\left(7_{4,3}-6_{4,2}\right) \mathrm{E}\left(v_{\mathrm{t}}=1\right)$ & 470 & 338.722 & $\mathrm{CH}_{3} \mathrm{OH}\left(7_{2,5}-6_{2,4}\right) \mathrm{E}$ & 87 \\
\hline 337.648 & $\mathrm{H}\left(7_{5,3}-6_{5,2}\right) \mathrm{E}\left(v_{\mathrm{t}}=1\right)$ & 611 & 338.723 & $\mathrm{CH}_{3} \mathrm{OH}\left(7_{2,6}-6_{2,5}\right) \mathrm{E}$ & 91 \\
\hline \multirow[t]{2}{*}{337.655} & $\mathrm{CH}_{3} \mathrm{OH}\left(7_{3,5}-6_{3,4}\right) \mathrm{A}\left(v_{\mathrm{t}}=1\right)$ & 461 & 338.760 & ${ }^{13} \mathrm{CH}_{3} \mathrm{OH}\left(13_{7,7}-12_{7,6}\right) \mathrm{A}$ & 206 \\
\hline & $\mathrm{CH}_{3} \mathrm{OH}\left(7_{3,4}-6_{3,3}\right) \mathrm{A}^{-}\left(v_{\mathrm{t}}=1\right)$ & 461 & 338.769 & $\mathrm{HC}_{3} \mathrm{~N}(37-36) v_{7}=2$ & 525 \\
\hline 337.671 & $\mathrm{CH}_{3} \mathrm{OH}\left(7_{2,6}-6_{2,5}\right) \mathrm{E}\left(v_{\mathrm{t}}=1\right)$ & 465 & 338.886 & $\mathrm{C}_{2} \mathrm{H}_{5} \mathrm{OH}\left(15_{7,8}-15_{6,19}\right)$ & 162 \\
\hline \multirow[t]{3}{*}{337.686} & $\mathrm{CH}_{3} \mathrm{OH}\left(7_{4,3}-6_{4,2}\right) \mathrm{A}\left(v_{\mathrm{t}}=1\right)$ & 546 & 339.058 & $\mathrm{C}_{2} \mathrm{H}_{5} \mathrm{OH}\left(14_{7,7}-14_{6,8}\right)$ & 150 \\
\hline & $\mathrm{CH}_{3} \mathrm{OH}\left(7_{4,4}-6_{4,3}\right) \mathrm{A}^{-}\left(v_{\mathrm{t}}=1\right)$ & 546 & 347.232 & $\mathrm{CH}_{2} \mathrm{CHCN}\left(38_{1,38}-37_{1,37}\right)$ & 329 \\
\hline & $\mathrm{CH}_{3} \mathrm{OH}\left(7_{5,2}-6_{5,1}\right) \mathrm{E}\left(v_{\mathrm{t}}=1\right)$ & 494 & 347.331 & ${ }^{28} \mathrm{SiO}(8-7)$ & 75 \\
\hline 337.708 & $\mathrm{CH}_{3} \mathrm{OH}\left(7_{1,6}-6_{1,5}\right) \mathrm{E}\left(v_{\mathrm{t}}=1\right)$ & 489 & 347.446 & UL & \\
\hline 337.722 & $\mathrm{CH}_{3} \mathrm{OCH}_{3}\left(7_{4,4}-6_{3,3}\right) \mathrm{EE}$ & 48 & 347.494 & $\mathrm{HCOOCH}_{3}\left(27_{5,22}-26_{5,21}\right) \mathrm{A}$ & 247 \\
\hline 337.732 & $\mathrm{CH}_{3} \mathrm{OCH}_{3}\left(7_{4,3}-6_{3,3}\right) \mathrm{EE}$ & 48 & 347.759 & $\mathrm{CH}_{2} \mathrm{CHCN}\left(36_{2,34}-35_{2,32}\right)$ & 317 \\
\hline 337.749 & $\mathrm{CH}_{3} \mathrm{OH}\left(7_{0,7}-6_{0,6}\right) \mathrm{A}\left(v_{\mathrm{t}}=1\right)$ & 489 & 347.792 & UL & \\
\hline 337.778 & $\mathrm{CH}_{3} \mathrm{OCH}_{3}\left(7_{4,4}-6_{3,4}\right) \mathrm{EE}$ & 48 & 347.842 & $\mathrm{UL}$ & \\
\hline 337.787 & $\mathrm{CH}_{3} \mathrm{OCH}_{3}\left(7_{4,3}-6_{3,4}\right) \mathrm{AA}$ & 48 & 347.916 & $\mathrm{C}_{2} \mathrm{H}_{5} \mathrm{OH}\left(2 \mathrm{O}_{4,17}-19_{4,16}\right)$ & 251 \\
\hline 337.825 & $\mathrm{HC}_{3} \mathrm{~N}(37-36) v_{7}=1$ & 629 & 347.983 & UL & \\
\hline 337.838 & $\mathrm{CH}_{3} \mathrm{OH}\left(20_{6,14}-21_{5,16}\right) \mathrm{E}$ & 676 & 348.261 & $\mathrm{CH}_{3} \mathrm{CH}_{2} \mathrm{CN}\left(39_{2,37}-38_{2,36}\right)$ & 344 \\
\hline 337.878 & $\mathrm{CH}_{3} \mathrm{OH}\left(7_{1,6}-6_{1,5}\right) \mathrm{A}\left(v_{\mathrm{t}}=2\right)$ & 748 & 348.340 & $\mathrm{HN}^{13} \mathrm{C}(4-3)$ & 42 \\
\hline 337.969 & $\mathrm{CH}_{3} \mathrm{OH}\left(7_{1,6}-6_{1,5}\right) \mathrm{A}\left(v_{\mathrm{t}}=1\right)$ & 390 & 348.345 & $\mathrm{CH}_{3} \mathrm{CH}_{2} \mathrm{CN}\left(40_{2,39}-39_{2,38}\right)$ & 351 \\
\hline 338.081 & $\mathrm{H}_{2} \mathrm{CS}\left(10_{1,10}-9_{1,9}\right)$ & 102 & 348.532 & $\mathrm{H}_{2} \mathrm{CS}\left(10_{1,9}-9_{1,8}\right)$ & 105 \\
\hline 338.125 & $\mathrm{CH}_{3} \mathrm{OH}\left(7_{0,7}-6_{0,6}\right) \mathrm{E}$ & 78 & 348.910 & $\mathrm{HCOOCH}_{3}\left(28_{9,20}-27_{9,19}\right) \mathrm{E}$ & 295 \\
\hline 338.143 & $\mathrm{CH}_{3} \mathrm{CH}_{2} \mathrm{CN}\left(37_{3,34}-36_{3,33}\right)$ & 317 & 348.911 & $\mathrm{CH}_{3} \mathrm{CN}\left(19_{9}-18_{9}\right)$ & 745 \\
\hline 338.306 & $\mathrm{SO}_{2}\left(14_{4,14}-18_{3,15}\right)$ & 197 & 349.025 & $\mathrm{CH}_{3} \mathrm{CN}\left(19_{8}-18_{8}\right)$ & 624 \\
\hline 338.345 & $\mathrm{CH}_{3} \mathrm{OH}\left(7_{1,7}-6_{1,6}\right) \mathrm{E}$ & 71 & 349.107 & $\mathrm{CH}_{3} \mathrm{OH}\left(14_{1,13}-14_{0,14}\right)$ & 43 \\
\hline 338.405 & $\mathrm{CH}_{3} \mathrm{OH}\left(7_{6,2}-6_{6,1}\right) \mathrm{E}$ & 244 & & & \\
\hline
\end{tabular}

${ }^{a}$ The detection of this $\mathrm{CH}_{3} \mathrm{OH} v_{\mathrm{t}}=2$ line is doubtful since other close $v_{\mathrm{t}}=2$ lines with similar excitation temperatures were not detected.

of the G29.96-0.02 hot core. The 14 optically identified Trapezia discussed by Abt \& Corbally (2000) have mean radii to the furthest outlying member of $\sim 4 \times 10^{4} \mathrm{AU}$, with the largest radius of $\sim 5.4 \times 10^{5} \mathrm{AU}(\sim 2.6 \mathrm{pc})$, the approximate dimension of an open cluster. Therefore, the protostellar projected separations of the tentative proto-Trapezium candidate in G29.96-0.02 are significantly smaller than in typical optically visible Trapezia systems. A similar small size for a candidate Trapezium system has recently been reported for the multiple system in W3IRS5 (Megeath et al. 2005).

The small sizes of the proto-Trapezia in G29.96-0.02 and W3IRS5 may be attributed to their youth. During their upcoming evolution, these young system will expel most of the surrounding gas and dust envelope via the protostellar outflows and strong uv-radiation. Therefore, the whole gravitational potential of the system will decrease and the kinetic energy may dominate. Systems with positive total energy will globally expand and will eventually be observable as a larger-scale optical Trapezia systems (Ambartsumian 1955).

With the given data it is hard to estimate how massive the expected Trapezia stars are and will finally be at the end of their formation processes. The integrated hot core luminosity is estimated to be $\sim 10^{5} L_{\odot}$ (Cesaroni et al. 1994; Olmi et al. 2003), in contrast to the integrated luminosity of the whole region 
measured by the large IRAS beam of $\sim 10^{6} L_{\odot}$. Producing $10^{5} L_{\odot}$ requires either an $\mathrm{O} 7$ star or a few stars of comparable but lower masses. Nevertheless, the numbers imply that this Trapezium system should form at least one or more massive stars. Although the gas masses we derived from our dust continuum data (Table 2) are relatively low, that does not necessarily imply that their mass reservoir is restricted to these gas masses because it is possible that they may accrete additional gas from the larger-scale envelope that is filtered out by our observations. This scenario is predicted by the competitive accretion model for massive star formation (e.g., Bonnell et al. 2004). The fact that the gas masses we find for the four strongest submm sources are all similar allows to speculate that they may form about similar mass stars in the end, however, this cannot be proven by these data in more detail.

Assuming that the projected size of the potential proto-Trapezium system in G29.96-0.02 of approximately $7800(\mathrm{AU})^{2}$ resembles a 3-dimensional sphere of radius $\sim 3900 \mathrm{AU}$, we can estimate the current protostellar volume density of the region to approximately $1.4 \times 10^{5}$ protostars per cubic pc. This number is larger than typical stellar densities in young clusters of the order $10^{4}$ stars per cubic pc (Lada \& Lada 2003), but it is still below the extremely high (proto)stellar densities required for protostellar merger models of the order $10^{6}$ to $10^{8}$ stars per cubic pc (Bonnell et al. 1998, 2004; Stahler et al. 2000; Bally \& Zinnecker 2005).

Although we have not yet observed the extremely high (proto)stellar densities predicted by the coalescence scenario, as soon as we observe massive star-forming regions with a spatial resolution $\leq 4000 \mathrm{AU}$, we begin to resolve multiplicity and potential proto-Trapezia (see also the recent observations of NGC 6334I and I(N) by Hunter et al. 2006). Furthermore, this (proto)stellar density may even be a lower limit, since we observe only a two-dimensional projection and are additionally sensitivity limited to masses $\geq 2.1 M_{\odot}$ (corresponding to the $3 \sigma$ flux limit of $63 \mathrm{mJy}$ beam $^{-1}$ at the assumed temperature of $100 \mathrm{~K})$. Higher spatial resolution has so far always increased the observed (proto)stellar densities, and it is possible that in the future we may reach the $10^{6}$ requirement for merging to play a role. However, it is also important to get better theoretical predictions of potential merger signatures that observers could look for.

\subsection{Various episodes of massive star formation?}

It is interesting to note that the previously identified mid-infrared source (De Buizer et al. 2002) is offset from the submm continuum peaks. Although the mid-infrared astrometry is usually relatively uncertain, the association of the mid-infrared peak with class II $\mathrm{CH}_{3} \mathrm{OH}$ maser emission with an absolute positional uncertainty of only 30 mas (Minier et al. 2001) is indicative that the offset may be real. Combining the facts that we find within a small region of only $\sim 20000 \mathrm{AU}(\sim 0.1 \mathrm{pc})$ at least three different regions of massive star formation - the UCHII region, the mid-infrared source, and the submm continuum sources - indicates that not all massive stars within the same evolving cluster are coeval but that sequences of massive star formation may take place even on such small spatial scales.

\subsection{Carbon mono-sulfide $C^{34} S$}

One of the most striking spectral line maps is from the rare carbon mono-sulfide isotopologue $\mathrm{C}^{34} \mathrm{~S}(7-6)$. Its emission peak is not toward the hot core nor any of the submm continuum peaks, but largely east of it in the interface region between the submm continuum peaks and the UCHII region. Hence, one likes to understand why the $\mathrm{C}^{34} \mathrm{~S}$ emission is that weak toward the hot core region and that strong at the hot core/UCHII region interface.

CS usually desorbs from dust grains at moderate temperatures of a few $10 \mathrm{~K}$, hence it should be observable relatively early in the evolution of a growing hot molecular core (e.g., Viti et al. 2004). From $100 \mathrm{~K}$ upwards $\mathrm{H}_{2} \mathrm{O}$ is released from grains, then it forms $\mathrm{OH}$ molecules, and the $\mathrm{OH}$ can react with $\mathrm{S}$ to $\mathrm{SO}$ and $\mathrm{SO}_{2}$ (e.g., Charnley 1997). Therefore, the initial high $\mathrm{CS}$ abundances should decrease with time while the $\mathrm{SO}$ and $\mathrm{SO}_{2}$ are expected to increase with time (e.g., Wakelam et al. 2005). As shown in Fig. $2,{ }^{34} \mathrm{SO}$ peaks toward the hot core where the derived $\mathrm{CH}_{3} \mathrm{OH}$ temperatures exceed the $\mathrm{H}_{2} \mathrm{O}$ evaporation temperature (see Sect. 4.4 and Fig. 6b), potentially validating this theoretical prediction. According to such chemical models, the hot core G29.96-0.02 should have a chemical age of at least a few times $10^{4}$ years.

The strong $\mathrm{C}^{34} \mathrm{~S}$ emission in the hot core/UCHII interface region may be explained in the same framework. In the molecular evolution scheme outlined above, one would expect low $\mathrm{C}^{34} \mathrm{~S}$ emission toward the hot core with a maybe symmetrical increase further-out. In the case of the G29.96-0.02 hot core, we have the decrease toward the center, but the emission rises only toward the east, north and west with the strongest increase in the eastern hot core/UCHII region interface. If one compares the $\mathrm{C}^{34} \mathrm{~S}$ morphology in Fig. 2 with the temperature distribution in Fig. 6b, one finds the lowest $\mathrm{CH}_{3} \mathrm{OH}$ temperatures right in the vicinity of the $\mathrm{C}^{34} \mathrm{~S}$ emission peaks, adding further support to the proposed chemical picture.

Extrapolating this scenario to other molecules, it indicates that species which are destroyed by $\mathrm{H}_{2} \mathrm{O}$, e.g., molecular ions such as $\mathrm{HCO}^{+}$or $\mathrm{N}_{2} \mathrm{H}^{+}$(e.g., Bergin et al. 1998), are no good probes of the inner regions of hot molecular cores.

\subsection{Temperature structure}

Leurini et al. (2004, 2007) investigated the diagnostic properties of methanol over a range of physical parameters typical of highmass star-forming regions. They found that the ground state lines of $\mathrm{CH}_{3} \mathrm{OH}$ are mainly tracers of the spatial density of the gas, although at submillimeter wavelengths high $k$ transitions are also sensitive to the kinetic temperature. However, in hot, dense regions such as hot cores, the effects of infrared pumping on the level populations due to the thermal heating of the dust is not negligible, but mimic the effect of collisional excitation. For the ground state line, Leurini et al. (2007) found that it is virtually impossible to distinguish between IR pumping and pumping by collisions, as both mechanisms equally populate the $v_{\mathrm{t}}=0$ levels. On the other hand, the vibrationally or torsionally excited lines have very high critical densities $\left(10^{10}-10^{11} \mathrm{~cm}^{-3}\right)$ and high level energies ( $T \geq 200 \mathrm{~K}$ ). They are difficult to be populated by collisions and trace the IR field instead.

To study the physical conditions of the gas around the main continuum peaks in G29.96-0.02, we analyzed only the emission coming from the $v_{\mathrm{t}}=1$ lines, as their optical depth is lower than for the ground state, and their emission is confined to the gas around the dust condensations, while the $v_{\mathrm{t}}=0$ transitions are more extended and can be affected by problems of missing flux. We first fitted the methanol emission of the $v_{t}=1$ lines (see Fig. 5) towards the peak position, using the method described by Leurini et al. $(2004,2007)$ that is based on an LVG analysis and includes radiative pumping (Leurini et al. 2007). The continuum emission derived in Sect. 3.1 was used in the 


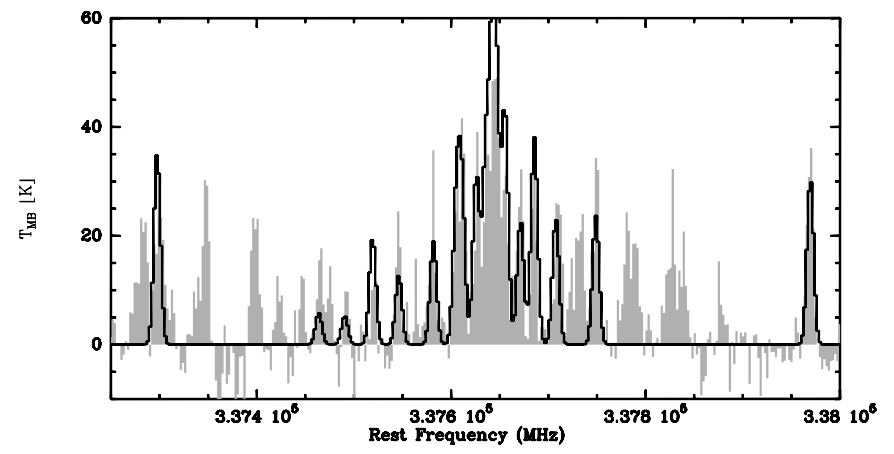

Fig. 5. Spectrum of the $7_{k_{a}, k_{b}} \rightarrow 6_{k_{a}, k_{b}-1} v_{\mathrm{t}}=1$ methanol band toward the main dust condensation. Overlaid in black is the synthetic spectrum resulting from the fit.

calculations to solve the equations for the level populations. The two main dust condensations submm 1 and submm 2 fall in the beam of the line data; however, we assumed that the emission is coming from only one component, which is more extended than our beam, and derived a $\mathrm{CH}_{3} \mathrm{OH}$ column density averaged over the beam of $4 \times 10^{17} \mathrm{~cm}^{-2}$. The corresponding methanol abundance, relative to $\mathrm{H}_{2}$ is of the order of $10^{-7}$, typical of hot core sources. Since the emission from the $v_{\mathrm{t}}=1$ lines is optically thin for this column density, and also at higher values, we consider this approach valid. The temperature derived toward the line peak is $340 \mathrm{~K}$. This corresponds to our best fit model, but from a $\chi^{2}$ analysis we can only infer a low limit of $\sim 220 \mathrm{~K}$ for the temperature of the gas. Since lines are optically thin, the degeneracy between kinetic temperature and column density is not solved, and the model delivers good fits to the $v_{\mathrm{t}}=1$ lines for lower or higher temperatures by adjusting the methanol column density. However, the low temperature solutions $\left(T_{\text {kin }}=100-200 \mathrm{~K}\right)$ need high methanol abundances relative to $\mathrm{H}_{2}\left(\sim 10^{-6}\right)$, which can be hardly found at these temperatures. Moreover, lines are optically thick for these column densities, and the assumption of our analysis is not valid anymore.

We also investigated the line ratio between several $v_{\mathrm{t}}=1$ lines at the column density derived for the main position, to find the best temperature diagnostic tool among the methanol lines and derive a temperature map of the region. We found that the line ratios with the blend of lines at $\sim 337.64 \mathrm{GHz}$ increase with the temperature of the gas (Fig. 6a). However, the blending of several transitions together complicates the use of such diagnostic. In Fig. 6b, we show the map of the line ratio between the $7_{1,6} \rightarrow 6_{1,5}-E v_{\mathrm{t}}=1$ at $337.708 \mathrm{GHz}$ and the blend between the $7_{1,7} \rightarrow 6_{1,6}-E v_{\mathrm{t}}=1$ at $337.642 \mathrm{GHz}$ and $7_{0,7} \rightarrow 6_{0,6}-E v_{\mathrm{t}}=1$ at $337.644 \mathrm{GHz}$. Since line intensities do not simply add up, we did not correct for the overlapping between the two transitions. Two other lines, the $7_{4,3} \rightarrow 6_{4,2}-E v_{\mathrm{t}}=1$ and the $7_{5,3} \rightarrow 6_{4,2}-E v_{\mathrm{t}}=1$, are also very close in frequency. This is seen in the linewidth of the blending, which is wider than for the other lines. Therefore, we considered only half of the channels of the blending at $337.64 \mathrm{GHz}$ in our line ratio analysis. From the ratio-map in Fig. 6b, submm1, submm2 and submm3 of Table 1 show high temperatures $(T \geq 300 \mathrm{~K})$, while relatively low temperature gas $(T \sim 100 \mathrm{~K})$ is found at RA [J2000] = $18^{\mathrm{h}} 46^{\mathrm{m}} 03^{\mathrm{s}} .818$ Dec $[\mathrm{J} 2000]=-02^{\circ} 39^{\prime} 22^{\prime \prime} .14$, close to a secondary peak of many ground state lines of methanol (Fig. 2). The temperature then decreases towards submm4. The increase in the line ratio towards the south-east and north is probably not true, but due to the poor signal to noise ratio in these areas. Changes in the column densities along the area may affect our results.

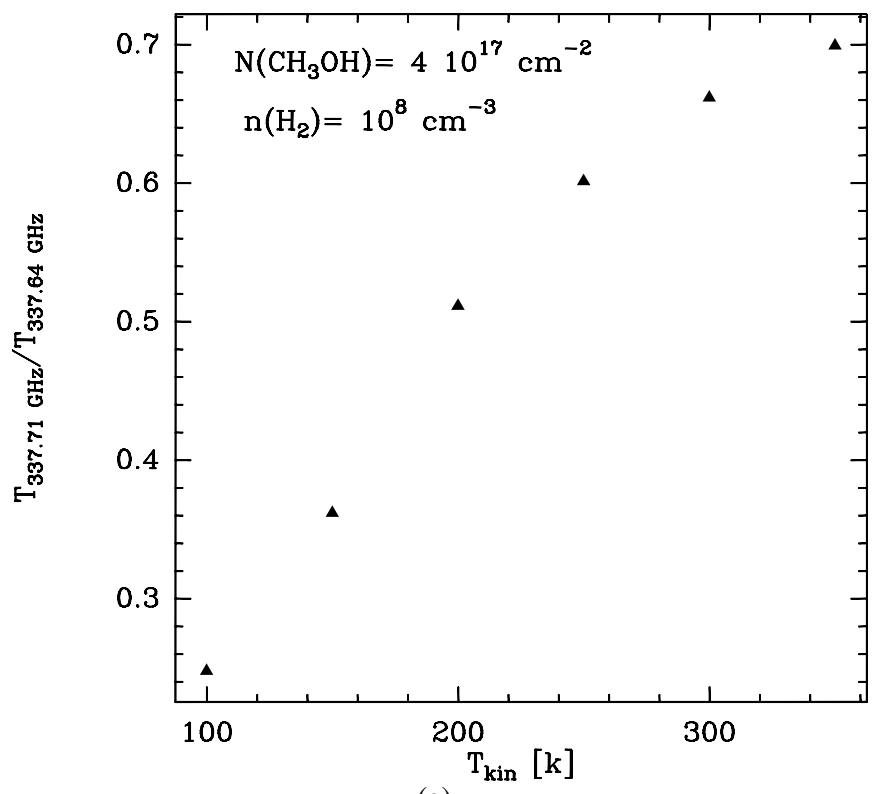

(a)

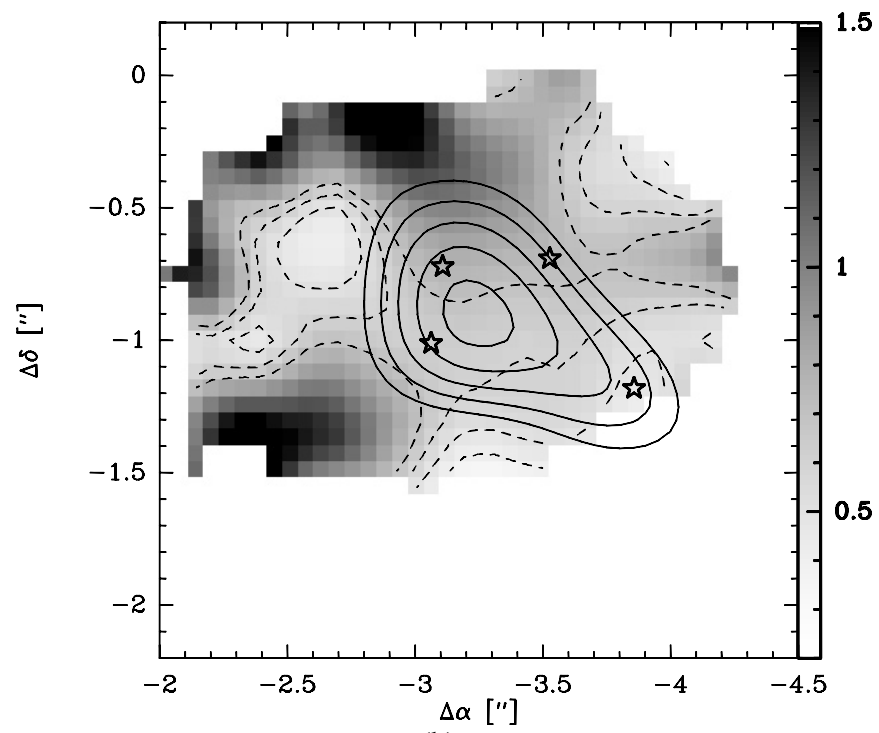

(b)

Fig. 6. a) Modeled line ratio between the $7_{1,6} \rightarrow 6_{1,5}-E v_{\mathrm{t}}=1$ line and the $7_{1,7} \rightarrow 6_{1,6}-E v_{\mathrm{t}}=1$ transitions, as function of the temperature. b) Map of the line ratio between the same transitions in the inner region around the peaks. The stars mark the positions of the dust peaks; the dashed contours show the values of the line ratio from $\sim 150$ to $\sim 350 \mathrm{~K}$, which correspond to levels from 0.3 to 0.7 in step of 0.1 in the map. The solid contours show the continuum emission smoothed to the resolution of the line data (from 0.2 to $0.4 \mathrm{Jy} /$ beam in step of 0.05 ). The offsets on the axes are relative to the phase center.

\subsection{Tracing rotation toward the massive cores}

At the given lower spatial resolution of the spectral line data compared to the submm continuum, we cannot resolve the four submm peaks well. However, one of the aims of such multi-line studies is to identify spectral lines that trace the massive protostars and that are potentially associated with massive disk-like structures. Such lines may then be used for kinematic gas studies of rotating gas envelopes, tori or accretion disks. Therefore, we analyzed the data-cubes searching for velocity structures indicative of any kind of rotation. In the large majority of spectral lines, this was not successful and we could mostly not identify 


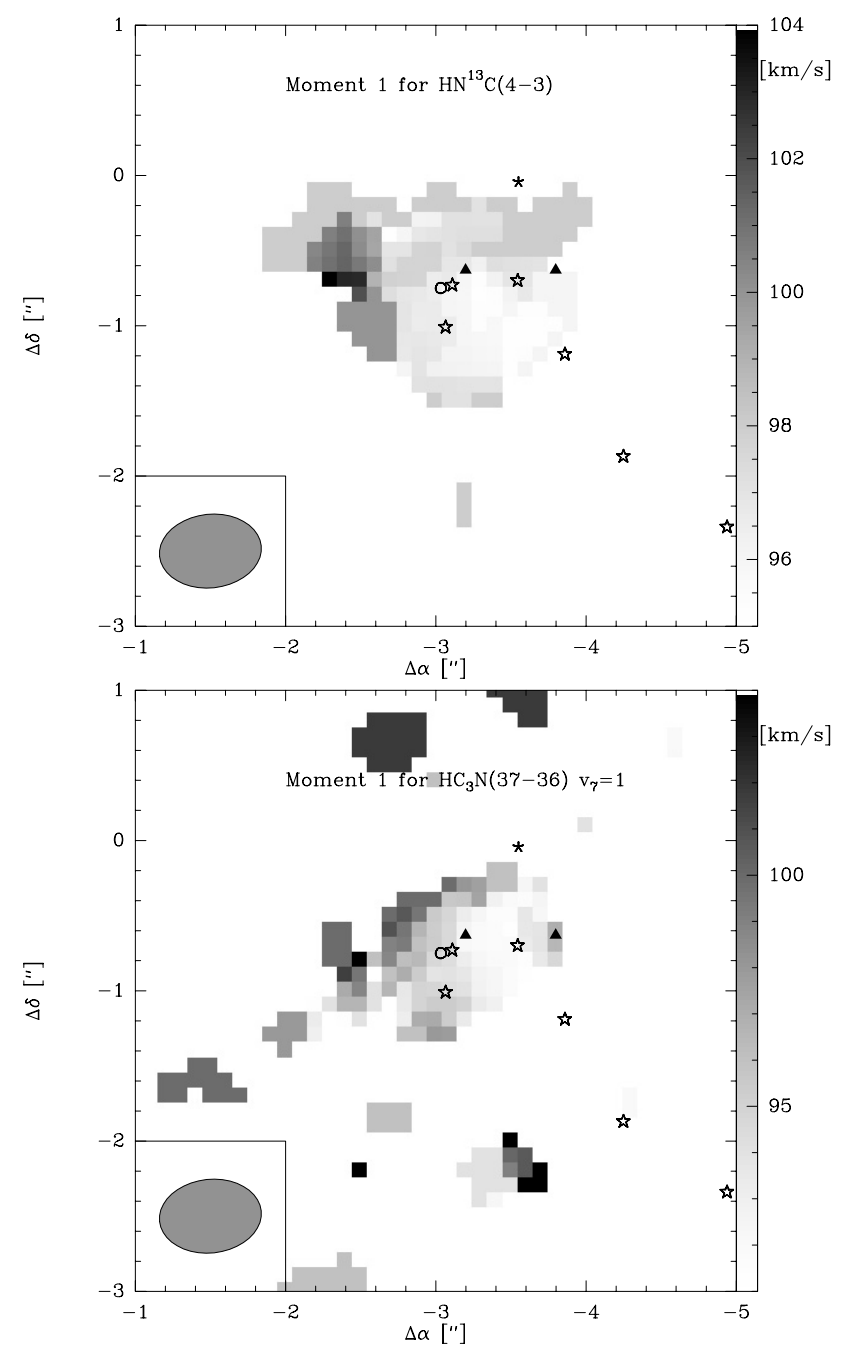

Fig. 7. Moment 1 maps of $\mathrm{HN}^{13} \mathrm{C}(4-3)$ (top) and $\mathrm{HC}_{3} \mathrm{~N}(37-36) v_{7}=1$ (bottom). The markers are the same as in the previous images, and the synthesized beam of $0.68^{\prime \prime} \times 0.49^{\prime \prime}$ is shown at the bottom left. The offsets on the axes are relative to the phase center.

coherent velocity structure. While chemical and temperature effects (Sects. 4.3 and 4.4) may be responsible for parts of that, the large column densities derived in Sect. 3.1 imply also large molecular line column densities and hence large optical depths. Therefore, many of the observed lines are likely optically thick tracing only outer gas layers of the hot molecular core not penetrating down to the deeply embedded protostars. Furthermore, many molecules would not only be excited in the central rotating disk-like structures but also in the surrounding envelope and maybe the outflow. Hence, disentangling the different components observationally remains a challenging task.

The major exceptions are the molecular lines of the rare isotopologue of hydrogen isocyanide $\mathrm{HN}^{13} \mathrm{C}(4-3)$ with a low excitation temperature of only $42 \mathrm{~K}$, and the vibrationally excited line of cyanoacetylene $\mathrm{HC}_{3} \mathrm{~N}(37-36) v_{7}=1$ with a higher excitation temperature of $629 \mathrm{~K}$ (Fig. 7). In both cases we find a velocity gradient across the main submm peak submm1 with a position angle of $\sim 45^{\circ}$ from north. This is approximately perpendicular to the molecular outflow discussed in Sect. 3.3 and by Gibb et al. (2004). Interestingly, Gibb et al. (2004) also find a similar velocity gradient in their central velocity channels of $\mathrm{H}_{2} \mathrm{~S}$. The previously reported $\mathrm{NH}_{3}$ and $\mathrm{CH}_{3} \mathrm{CN}$ velocity gradients in approximately east-west direction (Cesaroni et al. 1998;
Olmi et al. 2003) have been observed with slightly lower spatial resolution and are consistent with our data as well.

Our observations as well as previous work in the literature suggest that the G29.96-0.02 hot core exhibits a velocity gradient in the dense gas in approximately north-east south-west direction perpendicular to the molecular outflow observed at larger scales. Based on the $\mathrm{HN}^{13} \mathrm{C}(4-3)$ map, the diameter of this structure is $\sim 1.6^{\prime \prime}$ corresponding to radius of $\sim 4800$ AU. Since this emission encompasses not only the submm peak submm1 but also submm 2 and submm3, it is not genuine protostellar disk as often observed in low-mass star-forming regions. The velocity structure does not resemble Keplerian rotation and may hence be due to some larger-scale rotating envelope or torus that could transform into a genuine accretion disks at smaller still unresolved spatial scales (Cesaroni et al. 2007). Additional options to explain such a velocity gradient may be (a) interaction with the 2 nd outflow in north-east-south-western direction, (b) interaction with the expanding UCHII region, and (c) global collapse like recently proposed for NGC 2264 (Peretto et al. 2006). While we cannot exclude (a) and (b), option (c) of a globally collapsing core appears particularly interesting because combining rotation and collapse would result in an inward spiraling kinematic structure, potentially similar to the models originally proposed for rotating low-mass cores (e.g., Ulrich 1976; Terebey et al. 1984). Recent hydrodynamic simulations by Dobbs et al. (2005) and Krumholz et al. (2006) as well as analytic studies by Kratter \& Matzner (2006) find fragmentation and star formation within the massive disks forming early in the collapse process of highmass cores. This would be consistent with the found three subsources (submm1 to submm3) within the $\mathrm{HN}^{13} \mathrm{C} / \mathrm{HC}_{3} \mathrm{~N}$ structure. However, on a cautionary note it needs to be stressed that the collapse/rotation scenario is far from conclusive, and that the outflow and/or UCHII region can potentially influence the observed velocity pattern as well. It remains puzzling that only these two lines exhibit the discussed signatures whereas all the other spectral lines in our setup do not.

\section{Conclusions and summary}

The new $862 \mu \mathrm{m}$ submm continuum and spectral line data obtained with the SMA toward G29.96-0.02 at sub-arcsecond spatial resolution resolve the hot molecular core into several subsources. At an angular resolution of $0.36^{\prime \prime} \times 0.25^{\prime \prime}$, corresponding to linear scales of $\sim 1800 \mathrm{AU}$, the central core contains four submm continuum peaks which resemble a Trapezium-like multiple system at a very early evolutionary stage. Assuming spherical symmetry for the hot core region, the protostellar densities are high of the order $1.4 \times 10^{5}$ protostars per $\mathrm{pc}^{3}$. However, these protostellar densities are still below the required values between $10^{6}$ to $10^{8}$ protostars $/ \mathrm{pc}^{3}$ to make coalescence of protostars a feasible process. Derived $\mathrm{H}_{2}$ column densities of the order a few $10^{24} \mathrm{~cm}^{-2}$ imply visual extinctions of a few 1000 . The existence of three sites of massive star formation in different evolutionary stages within a small region (the UCHII region, the midinfrared source, and the submm continuum sources) indicates that sequences of massive star formation may take place within the same evolving massive protocluster.

The $4 \mathrm{GHz}$ of observed bandpass reveal a plethora of approximately 80 spectral lines from 18 molecular species, isotopologues or vibrationally excited lines. Only about $5 \%$ of the spectral lines remain unidentified. Most spectral lines peak toward the hot molecular core, while a few species also show more extended emission, likely due to molecular outflows and chemical differentiation. The $\mathrm{CH}_{3} \mathrm{OH}$ line forest allows us to investigate 
the temperature structure in more detail. We find hot core temperatures $\geq 300 \mathrm{~K}$ and decreasing temperature gradients to the core edges. The $\mathrm{SiO}(8-7)$ observations confirm a previously reported outflow Gibb et al. (2004) in north-west south-east direction with a potential identification of a second outflow emanating approximately in perpendicular direction. Furthermore, $\mathrm{C}^{34} \mathrm{~S}$ exhibits a peculiar morphology being weak toward the hot molecular core and strong in its surroundings, particular in the UCHII/hot core interface region. The $\mathrm{C}^{34} \mathrm{~S}$ deficiency toward the hot molecular core may be explained by time-dependent chemical desorption from grains, where the $\mathrm{C}^{34} \mathrm{~S}$ desorbs early, and later-on after $\mathrm{H}_{2} \mathrm{O}$ desorbs from grains forming $\mathrm{OH}$, the sulphur reacts with the $\mathrm{OH}$ to form $\mathrm{SO}$ and $\mathrm{SO}_{2}$.

Furthermore, we were interested in identifying the best molecular line tracers to investigate the kinematics and potential disk-like structures in such dense and young massive starforming regions. Most spectral lines do not exhibit any coherent velocity structure. A likely explanation for this uncorrelation between molecular line peaks and submm continuum peaks is that many spectral lines may be optically thick in such highcolumn-density regions, and that additional chemical evolution and temperature effects complicate the picture. Furthermore, many molecules are excited in various gas components (e.g., disk, envelope, outflow), and it is often observationally difficult to disentangle the different contributions properly. There are a few exceptions of optically thin and vibrationally excited lines that apparently probe deeper into the core tracing submm 1 better than other transitions. Investigating the velocity pattern of these spectral lines, we find for some of them a velocity gradient in the north-east south-west direction perpendicular to the molecular outflow. Since the spatial scale of this structure is relatively large ( $\sim 800 \mathrm{AU})$ comprising three of the central protostellar sources, and since the velocity structure is not Keplerian, this is not a genuine Keplerian accretion disk. While these data are consistent with a larger-scale toroid or envelope that may rotate and/or globally collapse, we cannot exclude other explanations, such as that the influence of the outflow(s) and/or expanding UCHII region produces the observed velocity pattern. In addition to this, these data confirm previous findings that the high column densities, the large optical depths of the spectral lines, the chemical evolution, and the different spectral line contributions from various gas components make it very difficult to identify suitable massive accretion disk tracers, and hence to study this phenomenon in a more statistical fashion. (e.g., Beuther et al. 2006)

Acknowledgements. We like to thank Peter Schilke and Sebastian Wolf for many interesting discussions about related subjects. We also thank the anonymous referee whose comments helped improving the paper. H.B. acknowledges financial support by the Emmy-Noether-Program of the Deutsche Forschungsgemeinschaft (DFG, grant BE2578).

\section{References}

Abt, H. A., \& Corbally, C. J. 2000, ApJ, 541, 841

Ambartsumian, V. A. 1955, The Observatory, 75, 72

Araya, E., Hofner, P., Goss, W. M., et al. 2006, ApJ, 643, L33

Bally, J., \& Zinnecker, H. 2005, AJ, 129, 2281

Bergin, E. A., Neufeld, D. A., \& Melnick, G. J. 1998, ApJ, 499, 777

Beuther, H., Schilke, P., Menten, K. M., et al. 2002, ApJ, 566, 945

Beuther, H., Schilke, P., Menten, K. M., et al. 2005a, ApJ, 633, 535

Beuther, H., Zhang, Q., Greenhill, L. J., et al. 2005b, ApJ, 632, 355

Beuther, H., Zhang, Q., Sridharan, T. K., Lee, C.-F., \& Zapata, L. A. 2006, A\&A, 454, 221

Bonnell, I. A., Bate, M. R., \& Zinnecker, H. 1998, MNRAS, 298, 93

Bonnell, I. A., Vine, S. G., \& Bate, M. R. 2004, MNRAS, 349, 735

Cesaroni, R., Churchwell, E., Hofner, P., Walmsley, C. M., \& Kurtz, S. 1994, A\&A, 288, 903

Cesaroni, R., Hofner, P., Walmsley, C. M., \& Churchwell, E. 1998, A\&A, 331, 709

Cesaroni, R., Galli, D., Lodato, G., Walmsley, C. M., \& Zhang, Q. 2007, in Protostars and Planets V, ed. B. Reipurth, D. Jewitt, \& K. Keil, 197

Charnley, S. B. 1997, ApJ, 481, 396

Churchwell, E., Walmsley, C. M., \& Cesaroni, R. 1990, A\&AS, 83, 119

De Buizer, J. M., Radomski, J. T., Piña, R. K., \& Telesco, C. M. 2002, ApJ, 580, 305

Dobbs, C. L., Bonnell, I. A., \& Clark, P. C. 2005, MNRAS, 360, 2

Frerking, M. A., Langer, W. D., \& Wilson, R. W. 1982, ApJ, 262, 590

Gibb, A. G., Wyrowski, F., \& Mundy, L. G. 2004, ApJ, 616, 301

Hatchell, J., Thompson, M. A., Millar, T. J., \& MacDonald, G. H. 1998, A\&AS, 133, 29

Hildebrand, R. H. 1983, QJRAS, 24, 267

Ho, P. T. P., Moran, J. M., \& Lo, K. Y. 2004, ApJ, 616, L1

Hoffman, I. M., Goss, W. M., Palmer, P., \& Richards, A. M. S. 2003, ApJ, 598, 1061

Hofner, P., \& Churchwell, E. 1996, A\&AS, 120, 283

Hunter, T. R., Brogan, C. L., Megeath, S. T., et al. 2006, ApJ, 649, 888

Kratter, K., \& Matzner, C. 2006, [arXiv:astro-ph/0609692]

Krumholz, M., Klein, R., \& McKee, C. 2006, [arXiv: astro-ph/0609798]

Lada, C. J., \& Lada, E. A. 2003, ARA\&A, 41, 57

Leurini, S., Schilke, P., Menten, K. M., et al. 2004, A\&A, 422, 573

Leurini, S., Schilke, P., Wyrowski, F., \& Menten, K. 2007, A\&A, 466, 215

Maxia, C., Testi, L., Cesaroni, R., \& Walmsley, C. M. 2001, A\&A, 371, 287

McCutcheon, W. H., Sandell, G., Matthews, H. E., et al. 2000, MNRAS, 316, 152

Megeath, S. T., Wilson, T. L., \& Corbin, M. R. 2005, ApJ, 622, L141

Minier, V., Conway, J. E., \& Booth, R. S. 2001, A\&A, 369, 278

Olmi, L., Cesaroni, R., Hofner, P., et al. 2003, A\&A, 407, 225

Peretto, N., Hennebelle, P., \& Andre, P. 2006, [arXiv: astro-ph/0611277]

Pratap, P., Megeath, S. T., \& Bergin, E. A. 1999, ApJ, 517, 799

Sault, R. J., Teuben, P. J., \& Wright, M. C. H. 1995, in Astronomical Data Analysis Software and Systems IV, ASP Conf. Ser., 77, 433

Schilke, P., Walmsley, C. M., Pineau des Forets, G., \& Flower, D. R. 1997, A\&A, 321,293

Scoville, N. Z., Carlstrom, J. E., Chandler, C. J., et al. 1993, PASP, 105, 1482 Sharpless, S. 1954, ApJ, 119, 334

Stahler, S. W., Palla, F., \& Ho, P. T. P. 2000, Protostars and Planets IV, 327

Terebey, S., Shu, F. H., \& Cassen, P. 1984, ApJ, 286, 529

Thompson, M. A., Hatchell, J., Walsh, A. J., MacDonald, G. H., \& Millar, T. J. 2006, A\&A, 453, 1003

Ulrich, R. K. 1976, ApJ, 210, 377

Viti, S., Collings, M. P., Dever, J. W., McCoustra, M. R. S., \& Williams, D. A. 2004, MNRAS, 354, 1141

Wakelam, V., Selsis, F., Herbst, E., \& Caselli, P. 2005, A\&A, 444, 883

Watson, A. M., \& Hanson, M. M. 1997, ApJ, 490, L165

Wood, D. O. S., \& Churchwell, E. 1989, ApJS, 69, 831

Wright, M. C. H., Plambeck, R. L., \& Wilner, D. J. 1996, ApJ, 469, 216

Wyrowski, F., Gibb, A. G., \& Mundy, L. G. 2002, in ASP Conf. Ser., 43 